CRYSTALLOGRAPHIC COMMUNICATIONS
Received 4 September 2016

Accepted 3 October 2016

Edited by M. Weil, Vienna University of Technology, Austria

Keywords: crystal structure; bis(imino)pyridine ligand; pyridine-diimine; redox-active; iron; radical anion.

CCDC reference: 1507934

Supporting information: this article has supporting information at journals.iucr.org/e

\section{Crystal structure of 1-phenylimido-1-\{6-[1-(phenyl- imino)ethyl]pyridin-2-yl\}ethan-1-yl- $\left.\kappa^{3} N, N^{\prime}, N^{\prime \prime}\right)$ - iron(II)}

\author{
Ka-Cheong Lau, Alexander S. Filatov and Richard F. Jordan*
}

Department of Chemistry, The University of Chicago, 5735 South Ellis ave, Chicago, Il 60637, USA. *Correspondence e-mail: rfjordan@uchicago.edu

The title iron complex, $\left[\mathrm{Fe}\left(\mathrm{C}_{21} \mathrm{H}_{19} \mathrm{~N}_{3}\right)_{2}\right]$, consists of an $\mathrm{Fe}^{\mathrm{II}}$ atom chelated by two tridentate bis(imino)pyridine radical anions in a slightly distorted octahedral coordination environment. In the solid state, there are two independent halfmolecules in the asymmetric unit, and the complete molecular structure is formed by applying twofold rotation symmetry with the twofold rotation axis passing through an $\mathrm{Fe}$ atom. In the crystal, the Fe-containing complexes are not involved in any particular direct intermolecular interactions, with the shortest $\mathrm{C}-\mathrm{H}_{\mathrm{Ar}}$ contacts between neighboring phenyl groups being $\mathrm{ca} 3.2 \AA$.

\section{Chemical context}

Transition metal complexes that contain bis(imino)pyridine ligands are highly active catalysts for olefin oligomerization and polymerization (Small et al., 1998; Britovsek et al., 1998, 1999; Small, 2015), and many other reactions (for example: Bart et al., 2004; Tondreau et al., 2012a,b; Obligacion \& Chirik, 2013; Bouwkamp et al., 2006; Hoyt et al., 2015; Sylvester \& Chirik, 2009). In pursuit of this chemistry, dicationic iron(II) complexes that are chelated by two neutral bis(imino)pyridine ligands have been synthesized and characterized by X-ray diffraction (for example: de Bruin et al., 2000; Ionkin et al., 2006). However, until recently, neutral \{bis(imino)pyridine $\}_{2} \mathrm{Fe}$ complexes were only generated in situ and characterized by cyclic voltammetry and electronic spectroscopy (de Bruin et al., 2000). Thus far, four neutral \{bis(imino)pyridine $\}_{2} \mathrm{Fe}$ complexes that contain alkyl or functionalizedphenyl substituents on the imine nitrogen atoms have been crystallographically characterized (Wile et al., 2009). Here we report the crystal structure of a parent molecule of the class, $(\mathrm{PDI})_{2} \mathrm{Fe}\left[\mathrm{PDI}=2,6-\left(\mathrm{C}_{6} \mathrm{H}_{5}-\mathrm{N}=\mathrm{CMe}\right)_{2}-\mathrm{C}_{5} \mathrm{H}_{3} \mathrm{~N}\right], \mathbf{1}$.

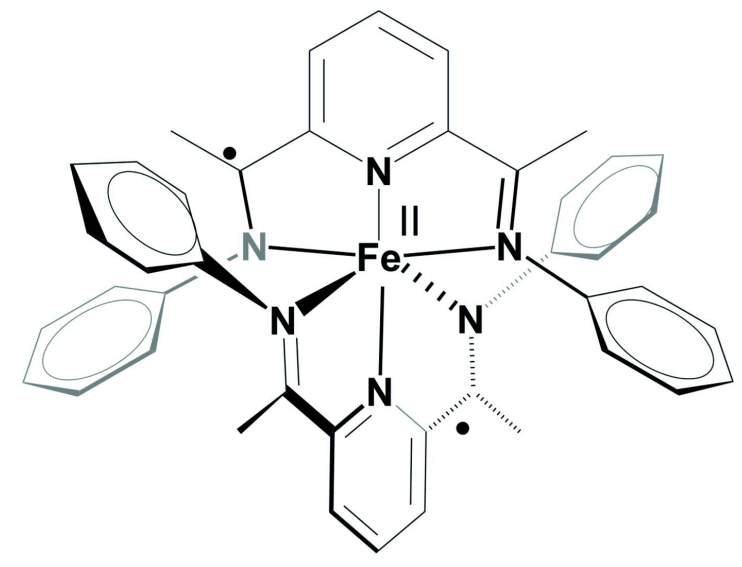

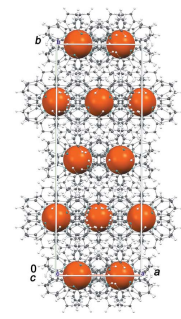




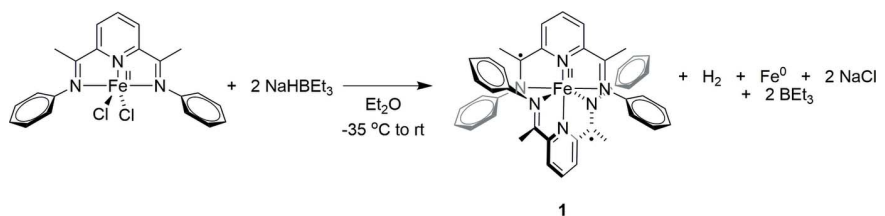

Figure 1

Schematic representation of the synthesis of $\mathbf{1}$.

\section{Structural commentary}

Complex 1 was synthesized by reduction of (PDI) $\mathrm{FeCl}_{2}$ with $\mathrm{NaHBEt}_{3}$ (Fig. 1). Crystals of $\mathbf{1}$ suitable for X-ray diffraction were obtained from $\mathrm{Et}_{2} \mathrm{O}$ solution. There are two independent molecules in the asymmetric unit (Fig. 2a). The whole molecular structure is formed by applying twofold rotation symmetry with the twofold rotation axis passing through an $\mathrm{Fe}$ atom (Fig. 2b). The two independent molecules have very similar bond lengths and angles except for the $\mathrm{N}$ (imine) $-\mathrm{Fe}$ bond lengths (Table 1$)$. One molecule (Fe2) has two equivalent $\mathrm{N}$ (imine) - Fe bond lengths [2.155 (2), 2.157 (2) ̊]], while the other (Fe1) has two noticeably different $\mathrm{N}$ (imine) $-\mathrm{Fe}$ bond lengths $[2.149$ (2), 2.173 (2) $⿱$ A] (Table 1). The C-C bond lengths in the pyridine and phenyl rings [1.380 (3)$1.401(3) \AA]$ and the $\mathrm{C}-\mathrm{N}$ bond lengths in the pyridine rings [1.366 (3), 1.372 (3) $\AA$ ] in the two molecules are very similar. The $\mathrm{N}$ (imine) $-\mathrm{Fe}-\mathrm{N}$ (pyridine) angles in the two molecules are also similar $\left[73.85(8)-75.01(8)^{\circ}\right]$. The two chelate planes formed by (PDI)Fe units are almost perpendicular to each other, presumably to avoid steric congestion [92.05 (9) and 93.32 (8) for Fe1- and Fe2-containing complexes, respectively, and measured as a dihedral angle between two planes passing through three nitrogen atoms of the coordinating PDI ligand].

An analogue of $\mathbf{1}$ containing a para-methoxy substituent on the imine-phenyl ring, $\left\{2,6-\left(4-\mathrm{MeO}-\mathrm{C}_{6} \mathrm{H}_{4}-\mathrm{N}=\mathrm{CMe}\right)_{2^{-}}\right.$ $\left.\mathrm{C}_{5} \mathrm{H}_{3} \mathrm{~N}\right\}_{2} \mathrm{Fe}$ (2) was also crystallized with two independent molecules in the asymmetric unit (Wile et al., 2009), and it is
Table 1

Selected bond lengths ( $)$.

\begin{tabular}{llll}
\hline $\mathrm{Fe} 1-\mathrm{N} 1$ & $2.149(2)$ & $\mathrm{N} 3-\mathrm{C} 14$ & $1.300(3)$ \\
$\mathrm{Fe} 1-\mathrm{N} 2$ & $2.028(2)$ & $\mathrm{C} 7-\mathrm{C} 9$ & $1.443(4)$ \\
$\mathrm{Fe} 1-\mathrm{N} 3$ & $2.173(2)$ & $\mathrm{C} 13-\mathrm{C} 14$ & $1.450(4)$ \\
$\mathrm{N} 1-\mathrm{C} 7$ & $1.327(3)$ & $\mathrm{Fe} 2-\mathrm{N} 4$ & $2.155(2)$ \\
$\mathrm{N} 2-\mathrm{C} 9$ & $1.368(3)$ & $\mathrm{Fe} 2-\mathrm{N} 5$ & $2.029(2)$ \\
$\mathrm{N} 2-\mathrm{C} 13$ & $1.372(3)$ & $\mathrm{Fe} 2-\mathrm{N} 6$ & $2.157(2)$ \\
\hline
\end{tabular}

interesting to compare the geometric parameters of $\mathbf{1}$ and $\mathbf{2}$. As observed for $\mathbf{1}$, the $\mathrm{N}$ (imine) $-\mathrm{Fe}$ bond lengths in one of the two independent molecules in the asymmetric unit of $\mathbf{2}$ are similar [2.1278 (19), 2.1481 (19) $\AA$ ], while those in the other exhibit much greater disparity [2.1159 (19), 2.1711 (19) $\AA$ ] . Although the electron-donating methoxy substituents of $\mathbf{2}$ are expected to render the imino nitrogens more basic than those in $\mathbf{1}$, the $\mathrm{N}$ (imine) - Fe bond lengths in $\mathbf{1}$ and $\mathbf{2}$ are very similar [range for 1: 2.149 (2) - 2.173 (2) $\AA$; range for 2: 2.1159 (19) $2.1711(19) \AA]$.

Bis(imino)pyridine ligands are redox-active owing to the extensive $\pi$-conjugation (de Bruin et al., 2000; Budzelaar et al., 2001; Knijnenburg et al., 2006). Reduction of the ligand causes characteristic changes in bond lengths, as expected from the resonance structures of the mono-reduced ligand as shown in Fig. 3 (Bart et al., 2006). In particular, reduction by $1 \mathrm{e}^{-}$ lengthens the $\mathrm{C}$ (imine) $-\mathrm{N}$ (imine) bond length from $\mathrm{ca} 1.28$ to $1.32 \AA$ and shortens the $\mathrm{C}(\mathrm{imine})-\mathrm{C}$ (ipso) bond length from ca 1.50 to $1.44 \AA$. In the free ligand, the C(imine) - N(imine) and $\mathrm{C}$ (imine)-C(ipso) bond lengths are 1.266 (4) and 1.497 (5) $\AA$ (Mentes et al., 2001). The electronic structure of 2 was shown to consist of an $\mathrm{Fe}^{\mathrm{II}}$ atom and two mono-reduced bis(imino)pyridine radical anions by Mössbauer spectroscopy, magnetic data, crystallographic data and broken-symmetry DFT calculations. The C(imine) $-\mathrm{N}$ (imine) [1.294 (3)1.327 (3) $\AA$ ] and C(imine) - C(ipso) [1.440 (4)-1.456 (3) $\AA$ ] bond lengths in $\mathbf{1}$ are close to those in $\mathbf{2}$ [C(imine) $-\mathrm{N}$ (imine) (a)

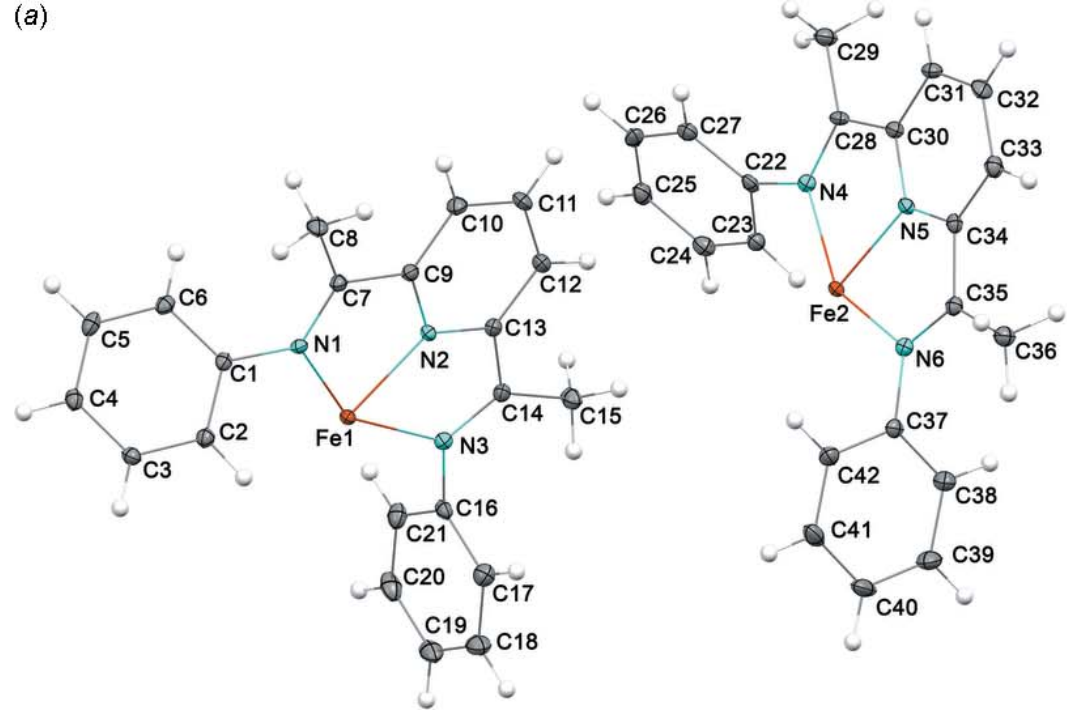

(b)

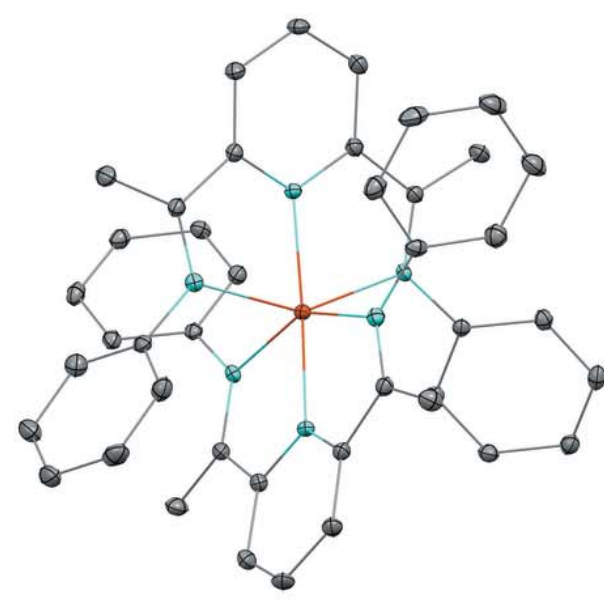

Figure 2

(a) The asymmetric unit of $\mathbf{1}$, showing the two half-complexes and (b) the molecular structure of one of the completed complexes (Fe1) with $\mathrm{H}$ atoms omitted for clarity and displacement ellipsoids shown at the $50 \%$ probability level. 


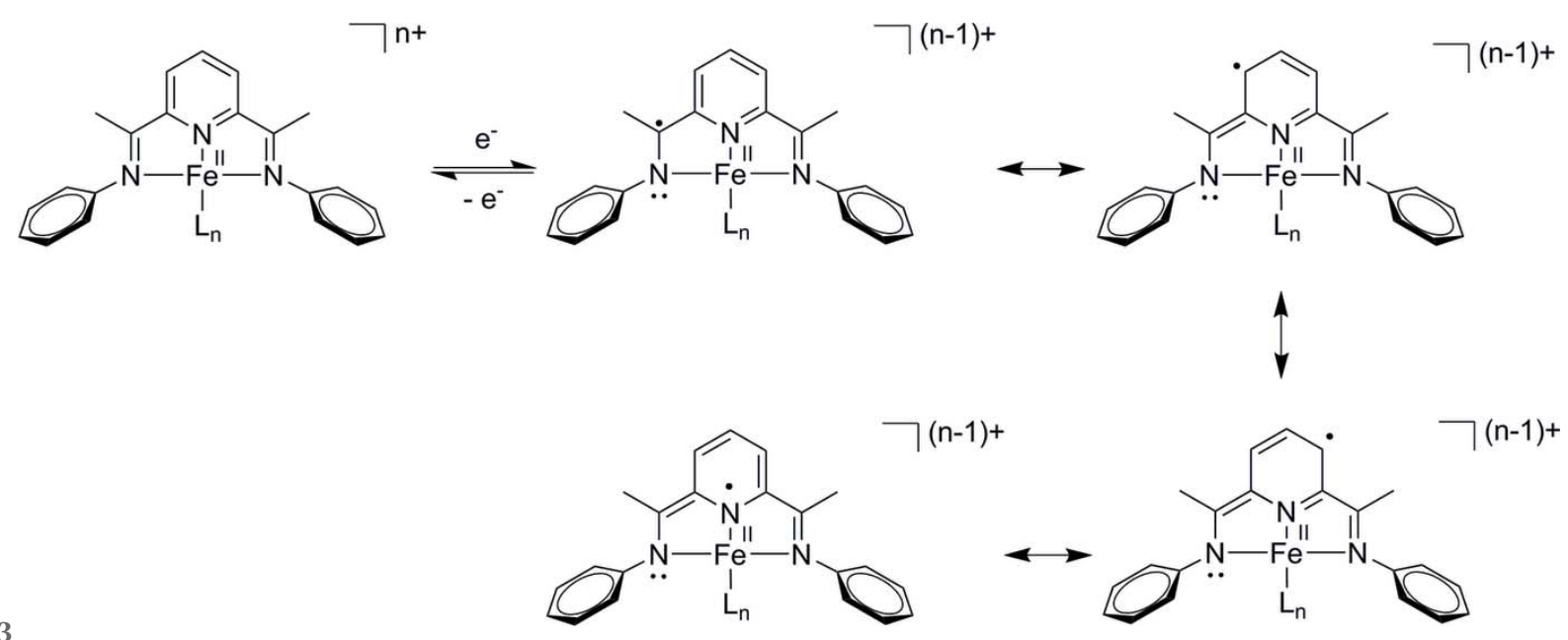

Figure 3

Resonance structures of the mono-reduced ligand in $\mathbf{1}$.

$=1.306$ (3) -1.313 (3) $\AA$ and $\mathrm{C}($ imine $)-\mathrm{C}(\mathrm{ipso})=1.432(3)-$ 1.444 (3) $\mathrm{]}$ ], consistent with mono-reduced PDI ligands and an $\mathrm{Fe}^{\mathrm{II}}$ atom as observed for $\mathbf{2}$.

\section{Supramolecular features}

The structure crystallizes in the orthorhombic Ccce space group (No. 68) with rather large unit-cell parameters ( $b$ and $c$ axes are both greater than $30 \AA$ ). Fig. 4 shows the crystal packing with $\mathrm{Fe}$ atoms forming a sub-lattice with $\simeq 1 / 4$ of the cell volume. The different relative orientation of ligands around the central $\mathrm{Fe}$ atoms leads to the obtained large unit cell. In the crystal, the Fe-containing complexes are not involved in any particular direct intermolecular interactions. The shortest $\mathrm{C} \cdots \mathrm{H}_{\mathrm{Ar}}$ contacts with neighboring phenyl groups start at about $3.2 \AA$.

\section{Database survey}

A search of the Cambridge Structural Database (CSD, Version 5.37, last update November 2015; Groom et al., 2016) reveals several crystallographically characterized neutral iron(II) complexes that are chelated by two bis(imino)pyridine radical anions [CSD refcodes: DUFCAJ, DUFBOW,
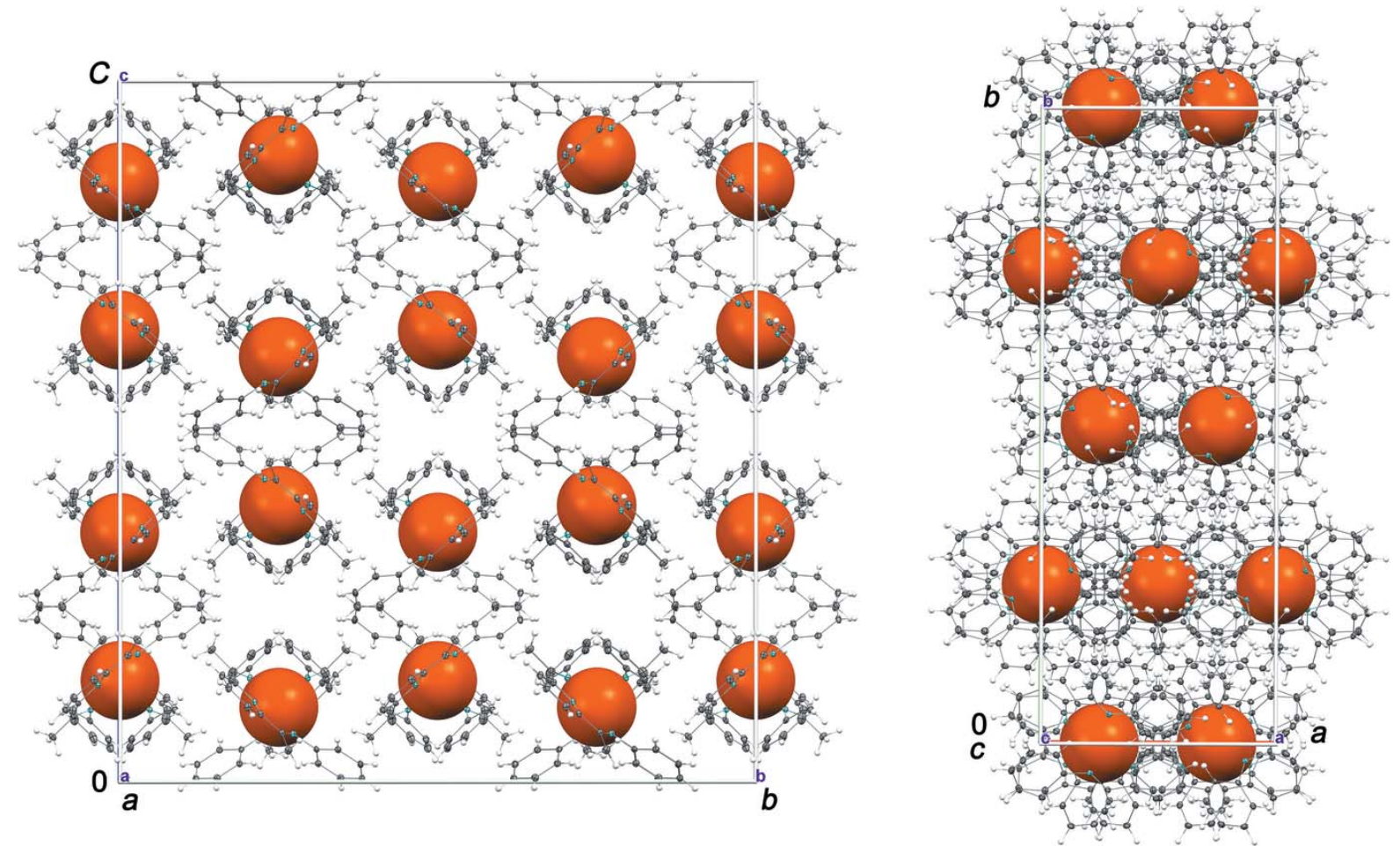

Figure 4

Orthogonal views of the crystal packing of $\mathbf{1}$ projected along the $a$ (left) and $c$ (right) axes. Fe atoms are shown as large brown spheres of arbitrary radius. 
DUFCEN, DUFBUC (Wile et al., 2009)]. Examples containing chromium [CSD refcode: OGUYOG (Wang et al., 2015)] and molybdenum [CSD refcode: OGUYEW (Wang et al., 2015)] have also been reported.

\section{Synthesis and crystallization}

Compound 1 was isolated from the attempted synthesis of (PDI)FeCl by reduction of (PDI)FeCl $\mathrm{Fi}_{2}$ with $\mathrm{NaHBEt}_{3}$ in $\mathrm{Et}_{2} \mathrm{O}$. $\mathrm{Et}_{2} \mathrm{O}(10 \mathrm{ml})$ was added to $(\mathrm{PDI}) \mathrm{FeCl}_{2}(0.113 \mathrm{~g}, 0.26 \mathrm{mmol})$ in a Schlenk flask to form a purple slurry. A solution of $\mathrm{NaHBEt}_{3}$ in $\mathrm{Et}_{2} \mathrm{O}(0.065 \mathrm{M}, 4 \mathrm{ml}, 0.26 \mathrm{mmol})$ was added dropwise at $238 \mathrm{~K}$ to the slurry. The mixture was warmed to room temperature ( $c a 293 \mathrm{~K}$ ) for $1 \mathrm{~h}$ and evolved to a red slurry. The mixture was filtered and the filtrate was concentrated under vacuum to afford purple crystals of $\mathbf{1}$, which were identified by $\mathrm{X}$-ray crystallographic analysis.

\section{Refinement}

Crystal data, data collection and structure refinement details are summarized in Table 2. All carbon-bound $\mathrm{H}$ atoms were included in idealized positions for structure factor calculations $\left[\mathrm{C}-\mathrm{H}=0.95-0.98 \AA, U_{\text {iso }}(\mathrm{H})\right.$ set to $\left.1.2-1.5 U_{\text {eq }}(\mathrm{C})\right]$.

\section{Acknowledgements}

This work was supported by the University of Chicago Women's Board.

\section{References}

Bart, S. C., Chłopek, K., Bill, E., Bouwkamp, M. W., Lobkovsky, E., Neese, F., Wieghardt, K. \& Chirik, P. J. (2006). J. Am. Chem. Soc. 128, 13901-13912.

Bart, S. C., Lobkovsky, E. \& Chirik, P. J. (2004). J. Am. Chem. Soc. 126, 13794-13807.

Bouwkamp, M. W., Bowman, A. C., Lobkovsky, E. \& Chirik, P. J. (2006). J. Am. Chem. Soc. 128, 13340-13341.

Britovsek, G. J. P., Bruce, M., Gibson, V. C., Kimberley, B. S., Maddox, P. J., Mastroianni, S., McTavish, S. J., Redshaw, C., Solan, G. A., Strömberg, S., White, A. J. P. \& Williams, D. J. (1999). J. Am. Chem. Soc. 121, 8728-8740.

Britovsek, G. J. P., Gibson, V. C., McTavish, S. J., Solan, G. A., White, A. J. P., Williams, D. J., Britovsek, G. J. P., Kimberley, B. S. \& Maddox, P. J. (1998). Chem. Commun. pp. 849-850.

Bruin, B. de, Bill, E., Bothe, E., Weyhermüller, T. \& Wieghardt, K. (2000). Inorg. Chem. 39, 2936-2947.

Bruker (2014). APEX2, SAINT, and SADABS. Bruker AXS Inc., Madison, Wisconsin, USA.

Budzelaar, P. H. M., de Bruin, B., Gal, A. W., Wieghardt, K. \& van Lenthe, J. H. (2001). Inorg. Chem. 40, 4649-4655.

Dolomanov, O. V., Bourhis, L. J., Gildea, R. J., Howard, J. A. K. \& Puschmann, H. (2009). J. Appl. Cryst. 42, 339-341.

Groom, C. R., Bruno, I. J., Lightfoot, M. P. \& Ward, S. C. (2016). Acta Cryst. B72, 171-179.

Hoyt, J. M., Schmidt, V. A., Tondreau, A. M. \& Chirik, P. J. (2015). Science, 349, 960-963.
Table 2

Experimental details.

\begin{tabular}{|c|c|}
\hline \multicolumn{2}{|l|}{ Crystal data } \\
\hline Chemical formula & {$\left[\mathrm{Fe}\left(\mathrm{C}_{21} \mathrm{H}_{19} \mathrm{~N}_{3}\right)_{2}\right]$} \\
\hline$M_{\mathrm{r}}$ & 682.63 \\
\hline Crystal system, space group & Orthorhombic, Ccce \\
\hline Temperature $(\mathrm{K})$ & 100 \\
\hline$a, b, c(\AA)$ & $\begin{array}{l}11.9028(5), 32.2189(14), \\
\quad 35.5223(15)\end{array}$ \\
\hline$V\left(\AA^{3}\right)$ & $13622.6(10)$ \\
\hline$Z$ & 16 \\
\hline Radiation type & Мo $K \alpha$ \\
\hline$\mu\left(\mathrm{mm}^{-1}\right)$ & 0.48 \\
\hline Crystal size $(\mathrm{mm})$ & $0.32 \times 0.24 \times 0.10$ \\
\hline \multicolumn{2}{|l|}{ Data collection } \\
\hline Diffractometer & $\begin{array}{l}\text { Bruker D8 Venture PHOTON } 100 \\
\text { CMOS }\end{array}$ \\
\hline Absorption correction & $\begin{array}{l}\text { Multi-scan (SADABS; Bruker, } \\
\text { 2014) }\end{array}$ \\
\hline$T_{\min }, T_{\max }$ & $0.662,0.745$ \\
\hline $\begin{array}{l}\text { No. of measured, independent and } \\
\text { observed }[I>2 \sigma(I)] \text { reflections }\end{array}$ & $116689,7006,5821$ \\
\hline$R_{\text {int }}$ & 0.062 \\
\hline$(\sin \theta / \lambda)_{\max }\left(\AA^{-1}\right)$ & 0.626 \\
\hline \multicolumn{2}{|l|}{ Refinement } \\
\hline$R\left[F^{2}>2 \sigma\left(F^{2}\right)\right], w R\left(F^{2}\right), S$ & $0.053,0.128,1.19$ \\
\hline No. of reflections & 7006 \\
\hline No. of parameters & 447 \\
\hline $\mathrm{H}$-atom treatment & $\mathrm{H}$-atom parameters constrained \\
\hline$\Delta \rho_{\max }, \Delta \rho_{\min }\left(\mathrm{e} \AA^{-3}\right)$ & $0.66,-0.63$ \\
\hline
\end{tabular}

Computer programs: APEX2 and SAINT (Bruker, 2014), SHELXT2014 (Sheldrick, 2015a), SHELXL2014 (Sheldrick, 2015b), OLEX2 (Dolomanov et al., 2009), Mercury (Macrae et al., 2008) and publCIF (Westrip, 2010).

Ionkin, A. S., Marshall, W. J., Adelman, D. J., Fones, B. B., Fish, B. M. \& Schiffhauer, M. F. (2006). Organometallics, 25, 2978-2992.

Knijnenburg, Q., Gambarotta, S. \& Budzelaar, P. H. M. (2006). Dalton Trans. pp. 5442-5448.

Macrae, C. F., Bruno, I. J., Chisholm, J. A., Edgington, P. R., McCabe, P., Pidcock, E., Rodriguez-Monge, L., Taylor, R., van de Streek, J. \& Wood, P. A. (2008). J. Appl. Cryst. 41, 466-470.

Mentes, A., Fawcett, J. \& Kemmitt, R. D. W. (2001). Acta Cryst. E57, o424-0425.

Obligacion, J. V. \& Chirik, P. (2013). Org. Lett. 15, 2680-2683.

Sheldrick, G. M. (2015a). Acta Cryst. A71, 3-8.

Sheldrick, G. M. (2015b). Acta Cryst. C71, 3-8.

Small, B. L. (2015). Acc. Chem. Res. 48, 2599-2611.

Small, B. L., Brookhart, M. \& Bennett, A. M. A. (1998). J. Am. Chem. Soc. 120, 4049-4050.

Sylvester, K. T. \& Chirik, P. J. (2009). J. Am. Chem. Soc. 131, 87728774.

Tondreau, A. M., Atienza, C. C. H., Darmon, J. M., Milsmann, C., Hoyt, H. M., Weller, K. J., Nye, S. A., Lewis, K. M., Boyer, J., Delis, J. G. P., Lobkovsky, E. \& Chirik, P. J. (2012b). Organometallics, 31, 4886-4893.

Tondreau, A. M., Atienza, C. C. H., Weller, K. J., Nye, S. A., Lewis, K. M., Delis, J. G. P. \& Chirik, P. J. (2012a). Science, 335, 567-570.

Wang, M., Weyhermüller, T. \& Wieghardt, K. (2015). Eur. J. Inorg. Chem. pp. 3246-3254.

Westrip, S. P. (2010). J. Appl. Cryst. 43, 920-925.

Wile, B. M., Trovitch, R. J., Bart, S. C., Tondreau, A. M., Lobkovsky, E., Milsmann, C., Bill, E., Wieghardt, K. \& Chirik, P. J. (2009). Inorg. Chem. 48, 4190-4200. 


\section{supporting information}

Acta Cryst. (2016). E72, 1595-1598 [https://doi.org/10.1107/S2056989016015528]

\section{Crystal structure of 1-phenylimido-1-\{6-[1-(phenylimino)ethyl]pyridin-2-} yl\}ethan-1-yl- $\left.\kappa^{3} N, N^{\prime}, N^{\prime \prime}\right)$ iron(II)

\section{Ka-Cheong Lau, Alexander S. Filatov and Richard F. Jordan}

Computing details

Data collection: APEX2 (Bruker, 2014); cell refinement: SAINT (Bruker, 2014); data reduction: SAINT (Bruker, 2014); program(s) used to solve structure: SHELXT2014 (Sheldrick, 2015a); program(s) used to refine structure: SHELXL2014 (Sheldrick, 2015b); molecular graphics: OLEX2 (Dolomanov et al., 2009) and Mercury (Macrae et al., 2008); software used to prepare material for publication: publCIF (Westrip, 2010).

1-Phenylimido-1-\{6-[1-(phenylimino)ethyl] pyridin-2-yl\}ethan-1-yl- $\left.\kappa^{3} N, N^{\prime}, N^{\prime \prime}\right)$ iron(II)

\section{Crystal data}

$\left[\mathrm{Fe}\left(\mathrm{C}_{21} \mathrm{H}_{19} \mathrm{~N}_{3}\right)_{2}\right]$

$M_{r}=682.63$

Orthorhombic, Ccce

$a=11.9028(5) \AA$

$b=32.2189(14) \AA$

$c=35.5223(15) \AA$

$V=13622.6(10) \AA^{3}$

$Z=16$

$F(000)=5728$

Data collection

Bruker D8 Venture PHOTON 100 CMOS diffractometer

Radiation source: INCOATEC ImuS microfocus source

Mirrors monochromator

Detector resolution: 10.4167 pixels $\mathrm{mm}^{-1}$

$\omega$ and phi scans

Absorption correction: multi-scan

(SADABS; Bruker, 2014)

Refinement

Refinement on $F^{2}$

Least-squares matrix: full

$R\left[F^{2}>2 \sigma\left(F^{2}\right)\right]=0.053$

$w R\left(F^{2}\right)=0.128$

$S=1.19$

7006 reflections

447 parameters

0 restraints

Primary atom site location: dual
$D_{\mathrm{x}}=1.331 \mathrm{Mg} \mathrm{m}^{-3}$

Mo $K \alpha$ radiation, $\lambda=0.71073 \AA$

Cell parameters from 9834 reflections

$\theta=2.2-26.4^{\circ}$

$\mu=0.48 \mathrm{~mm}^{-1}$

$T=100 \mathrm{~K}$

Plate, dark violet

$0.32 \times 0.24 \times 0.10 \mathrm{~mm}$

$T_{\min }=0.662, T_{\max }=0.745$

116689 measured reflections

7006 independent reflections

5821 reflections with $I>2 \sigma(I)$

$R_{\text {int }}=0.062$

$\theta_{\text {max }}=26.4^{\circ}, \theta_{\text {min }}=1.9^{\circ}$

$h=-14 \rightarrow 14$

$k=-40 \rightarrow 40$

$l=-44 \rightarrow 44$

Secondary atom site location: difference Fourier map

Hydrogen site location: inferred from neighbouring sites

$\mathrm{H}$-atom parameters constrained

$w=1 /\left[\sigma^{2}\left(F_{\mathrm{o}}^{2}\right)+(0.0365 P)^{2}+50.0482 P\right]$

where $P=\left(F_{\mathrm{o}}^{2}+2 F_{\mathrm{c}}{ }^{2}\right) / 3$

$(\Delta / \sigma)_{\max }=0.002$

$\Delta \rho_{\max }=0.66 \mathrm{e}^{-3}$

$\Delta \rho_{\min }=-0.63$ e $\AA^{-3}$ 


\section{Special details}

Geometry. All esds (except the esd in the dihedral angle between two 1.s. planes) are estimated using the full covariance matrix. The cell esds are taken into account individually in the estimation of esds in distances, angles and torsion angles; correlations between esds in cell parameters are only used when they are defined by crystal symmetry. An approximate (isotropic) treatment of cell esds is used for estimating esds involving l.s. planes.

Fractional atomic coordinates and isotropic or equivalent isotropic displacement parameters $\left(\hat{A}^{2}\right)$

\begin{tabular}{|c|c|c|c|c|}
\hline & $x$ & $y$ & $z$ & $U_{\text {iso }} * / U_{\text {eq }}$ \\
\hline $\mathrm{Fe} 1$ & 0.5000 & 0.7500 & $0.60614(2)$ & $0.01348(13)$ \\
\hline N1 & $0.37427(17)$ & $0.77288(6)$ & $0.56801(6)$ & $0.0146(4)$ \\
\hline $\mathrm{N} 2$ & $0.36620(17)$ & $0.71185(6)$ & $0.61341(6)$ & $0.0148(4)$ \\
\hline N3 & $0.55060(18)$ & $0.70451(6)$ & $0.64801(6)$ & $0.0166(5)$ \\
\hline $\mathrm{C} 1$ & $0.3933(2)$ & $0.80895(8)$ & $0.54604(7)$ & $0.0151(5)$ \\
\hline $\mathrm{C} 2$ & $0.4937(2)$ & $0.81186(8)$ & $0.52588(7)$ & $0.0189(6)$ \\
\hline $\mathrm{H} 2$ & 0.5457 & 0.7895 & 0.5264 & $0.023 *$ \\
\hline $\mathrm{C} 3$ & $0.5181(2)$ & $0.84716(8)$ & $0.50503(8)$ & $0.0195(6)$ \\
\hline $\mathrm{H} 3$ & 0.5861 & 0.8485 & 0.4911 & $0.023 *$ \\
\hline $\mathrm{C} 4$ & $0.4444(2)$ & $0.88047(8)$ & $0.50431(8)$ & $0.0205(6)$ \\
\hline H4 & 0.4614 & 0.9045 & 0.4899 & $0.025^{*}$ \\
\hline $\mathrm{C} 5$ & $0.3457(2)$ & $0.87820(8)$ & $0.52478(8)$ & $0.0220(6)$ \\
\hline H5 & 0.2952 & 0.9010 & 0.5247 & $0.026^{*}$ \\
\hline C6 & $0.3199(2)$ & $0.84291(8)$ & $0.54545(7)$ & $0.0183(5)$ \\
\hline H6 & 0.2517 & 0.8418 & 0.5593 & $0.022 *$ \\
\hline C7 & $0.2742(2)$ & $0.75478(8)$ & $0.56928(7)$ & $0.0169(5)$ \\
\hline $\mathrm{C} 8$ & $0.1756(2)$ & $0.76466(9)$ & $0.54468(8)$ & $0.0231(6)$ \\
\hline H8A & 0.1223 & 0.7820 & 0.5586 & $0.035^{*}$ \\
\hline H8B & 0.1386 & 0.7388 & 0.5370 & $0.035^{*}$ \\
\hline $\mathrm{H} 8 \mathrm{C}$ & 0.2014 & 0.7796 & 0.5223 & $0.035^{*}$ \\
\hline C9 & $0.2667(2)$ & $0.72135(8)$ & $0.59619(8)$ & $0.0174(5)$ \\
\hline $\mathrm{C} 10$ & $0.1672(2)$ & $0.70106(8)$ & $0.60645(8)$ & $0.0230(6)$ \\
\hline H10 & 0.0983 & 0.7080 & 0.5945 & $0.028^{*}$ \\
\hline C11 & $0.1700(2)$ & $0.67089(9)$ & $0.63407(9)$ & $0.0245(6)$ \\
\hline H11 & 0.1029 & 0.6572 & 0.6414 & $0.029 *$ \\
\hline $\mathrm{C} 12$ & $0.2715(2)$ & $0.66062(8)$ & $0.65106(8)$ & $0.0210(6)$ \\
\hline H12 & 0.2747 & 0.6393 & 0.6695 & $0.025^{*}$ \\
\hline $\mathrm{C} 13$ & $0.3675(2)$ & $0.68179(8)$ & $0.64075(7)$ & $0.0168(5)$ \\
\hline C14 & $0.4765(2)$ & $0.67718(8)$ & $0.65847(7)$ & $0.0174(5)$ \\
\hline $\mathrm{C} 15$ & 0.4957 (2) & $0.64459(9)$ & $0.68814(8)$ & $0.0243(6)$ \\
\hline H15A & 0.5765 & 0.6418 & 0.6929 & $0.036^{*}$ \\
\hline H15B & 0.4655 & 0.6180 & 0.6794 & $0.036^{*}$ \\
\hline $\mathrm{H} 15 \mathrm{C}$ & 0.4577 & 0.6528 & 0.7114 & $0.036^{*}$ \\
\hline C16 & $0.6627(2)$ & $0.70349(8)$ & $0.66146(7)$ & $0.0182(5)$ \\
\hline $\mathrm{C} 17$ & $0.7394(3)$ & $0.67610(9)$ & $0.64561(8)$ & $0.0255(6)$ \\
\hline H17 & 0.7143 & 0.6560 & 0.6279 & $0.031 *$ \\
\hline $\mathrm{C} 18$ & $0.8519(3)$ & $0.67774(10)$ & $0.65533(9)$ & $0.0319(7)$ \\
\hline H18 & 0.9033 & 0.6586 & 0.6445 & $0.038^{*}$ \\
\hline C19 & 0.8899 (3) & $0.70702(10)$ & $0.68070(10)$ & $0.0335(7)$ \\
\hline
\end{tabular}




\begin{tabular}{|c|c|c|c|c|}
\hline H19 & 0.9674 & 0.7084 & 0.6871 & $0.040^{*}$ \\
\hline $\mathrm{C} 20$ & $0.8139(3)$ & $0.73426(10)$ & $0.69674(9)$ & $0.0325(7)$ \\
\hline $\mathrm{H} 20$ & 0.8393 & 0.7543 & 0.7144 & $0.039 *$ \\
\hline $\mathrm{C} 21$ & 0.7009 (3) & $0.73262(9)$ & $0.68734(8)$ & $0.0264(6)$ \\
\hline $\mathrm{H} 21$ & 0.6495 & 0.7515 & 0.6986 & $0.032 *$ \\
\hline $\mathrm{Fe} 2$ & 0.2500 & 0.5000 & $0.64429(2)$ & 0.01289 (13 \\
\hline N4 & $0.12861(18)$ & $0.52706(6)$ & $0.68203(6)$ & 0.0157 (4) \\
\hline N5 & $0.10938(18)$ & $0.46510(6)$ & $0.63800(6)$ & $0.0146(4)$ \\
\hline N6 & $0.29045(18)$ & $0.45369(6)$ & $0.60258(6)$ & $0.0160(4)$ \\
\hline $\mathrm{C} 22$ & $0.1573(2)$ & $0.56140(8)$ & $0.70498(7)$ & $0.0165(5)$ \\
\hline $\mathrm{C} 23$ & $0.2584(2)$ & $0.55886(8)$ & $0.72503(7)$ & $0.0188(5)$ \\
\hline $\mathrm{H} 23$ & 0.3041 & 0.5348 & 0.7225 & $0.023 *$ \\
\hline $\mathrm{C} 24$ & $0.2929(2)$ & $0.59069(8)$ & $0.74837(8)$ & $0.0225(6)$ \\
\hline $\mathrm{H} 24$ & 0.3605 & 0.5879 & 0.7623 & $0.027 *$ \\
\hline $\mathrm{C} 25$ & $0.2294(2)$ & $0.62673(9)$ & $0.75159(8)$ & $0.0236(6)$ \\
\hline $\mathrm{H} 25$ & 0.2529 & 0.6486 & 0.7677 & $0.028 *$ \\
\hline $\mathrm{C} 26$ & $0.1311(2)$ & $0.63013(8)$ & $0.73093(8)$ & $0.0229(6)$ \\
\hline H26 & 0.0877 & 0.6548 & 0.7326 & $0.027 *$ \\
\hline $\mathrm{C} 27$ & $0.0946(2)$ & $0.59791(8)$ & $0.70769(8)$ & $0.0201(6)$ \\
\hline $\mathrm{H} 27$ & 0.0271 & 0.6008 & 0.6937 & $0.024 *$ \\
\hline $\mathrm{C} 28$ & $0.0248(2)$ & $0.51224(8)$ & $0.68000(7)$ & $0.0163(5)$ \\
\hline C29 & $-0.0753(2)$ & $0.52709(9)$ & $0.70198(8)$ & $0.0252(6)$ \\
\hline H29A & -0.0498 & 0.5432 & 0.7238 & $0.038^{*}$ \\
\hline H29B & -0.1190 & 0.5031 & 0.7106 & $0.038^{*}$ \\
\hline $\mathrm{H} 29 \mathrm{C}$ & -0.1224 & 0.5446 & 0.6859 & $0.038^{*}$ \\
\hline C30 & $0.0117(2)$ & $0.47772(8)$ & $0.65463(7)$ & $0.0170(5)$ \\
\hline C31 & $-0.0902(2)$ & $0.45885(8)$ & $0.64529(8)$ & $0.0211(6)$ \\
\hline H31 & -0.1579 & 0.4679 & 0.6568 & $0.025 *$ \\
\hline C32 & $-0.0925(2)$ & $0.42691(9)$ & $0.61925(8)$ & $0.0241(6)$ \\
\hline H32 & -0.1617 & 0.4139 & 0.6129 & $0.029 *$ \\
\hline $\mathrm{C} 33$ & $0.0070(2)$ & $0.41386(8)$ & $0.60236(8)$ & $0.0212(6)$ \\
\hline H33 & 0.0071 & 0.3916 & 0.5848 & $0.025^{*}$ \\
\hline C34 & $0.1056(2)$ & $0.43407(8)$ & $0.61172(7)$ & 0.0159 (5) \\
\hline C35 & $0.2133(2)$ & $0.42730(8)$ & $0.59329(7)$ & $0.0161(5)$ \\
\hline C36 & 0.2259 (2) & $0.39396(9)$ & $0.56407(8)$ & $0.0223(6)$ \\
\hline $\mathrm{H} 36 \mathrm{~A}$ & 0.1913 & 0.4031 & 0.5405 & $0.033^{*}$ \\
\hline H36B & 0.1889 & 0.3686 & 0.5728 & $0.033^{*}$ \\
\hline $\mathrm{H} 36 \mathrm{C}$ & 0.3059 & 0.3884 & 0.5599 & $0.033^{*}$ \\
\hline C37 & $0.4006(2)$ & $0.45267(8)$ & $0.58710(7)$ & $0.0178(5)$ \\
\hline C38 & $0.4804(2)$ & $0.42575(9)$ & $0.60131(9)$ & $0.0264(6)$ \\
\hline H38 & 0.4595 & 0.4054 & 0.6194 & $0.032 *$ \\
\hline C39 & $0.5910(3)$ & $0.42826(10)$ & $0.58929(10)$ & $0.0360(8)$ \\
\hline H39 & 0.6451 & 0.4094 & 0.5990 & $0.043^{*}$ \\
\hline $\mathrm{C} 40$ & $0.6235(3)$ & $0.45784(10)$ & $0.56331(10)$ & $0.0362(8)$ \\
\hline $\mathrm{H} 40$ & 0.6997 & 0.4599 & 0.5556 & $0.043^{*}$ \\
\hline $\mathrm{C} 41$ & $0.5432(3)$ & $0.48453(10)$ & $0.54870(9)$ & $0.0324(7)$ \\
\hline H41 & 0.5642 & 0.5047 & 0.5305 & $0.039 *$ \\
\hline $\mathrm{C} 42$ & $0.4326(2)$ & $0.48196(9)$ & $0.56043(8)$ & $0.0233(6)$ \\
\hline
\end{tabular}


$0.028^{*}$

Atomic displacement parameters $\left(\AA^{2}\right)$

\begin{tabular}{|c|c|c|c|c|c|c|}
\hline & $U^{11}$ & $U^{22}$ & $U^{33}$ & $U^{12}$ & $U^{13}$ & $U^{23}$ \\
\hline $\mathrm{Fe} 1$ & $0.0106(2)$ & $0.0120(2)$ & $0.0179(3)$ & $-0.00086(19)$ & 0.000 & 0.000 \\
\hline N1 & $0.0102(10)$ & $0.0139(10)$ & $0.0197(11)$ & $0.0024(8)$ & $-0.0003(8)$ & $-0.0004(9)$ \\
\hline $\mathrm{N} 2$ & $0.0115(10)$ & $0.0120(10)$ & $0.0210(11)$ & $-0.0015(8)$ & $0.0005(8)$ & $-0.0009(8)$ \\
\hline N3 & $0.0149(11)$ & $0.0154(11)$ & $0.0194(11)$ & $0.0006(9)$ & $-0.0002(9)$ & $0.0012(9)$ \\
\hline $\mathrm{C} 1$ & $0.0151(13)$ & $0.0135(12)$ & $0.0166(12)$ & $-0.0002(10)$ & $-0.0038(10)$ & $-0.0016(10)$ \\
\hline $\mathrm{C} 2$ & $0.0190(13)$ & $0.0157(12)$ & $0.0218(14)$ & $0.0002(11)$ & $-0.0017(11)$ & $-0.0009(11)$ \\
\hline $\mathrm{C} 3$ & $0.0148(13)$ & $0.0206(13)$ & $0.0231(14)$ & $-0.0023(11)$ & $0.0020(11)$ & $-0.0007(11)$ \\
\hline $\mathrm{C} 4$ & 0.0219 (14) & $0.0163(13)$ & $0.0234(14)$ & $-0.0011(11)$ & $-0.0051(11)$ & $0.0033(11)$ \\
\hline $\mathrm{C} 5$ & $0.0268(15)$ & $0.0160(13)$ & $0.0231(14)$ & $0.0051(11)$ & $-0.0076(12)$ & $-0.0016(11)$ \\
\hline C6 & $0.0173(13)$ & $0.0180(13)$ & $0.0195(13)$ & $0.0021(11)$ & $-0.0031(10)$ & $-0.0023(10)$ \\
\hline $\mathrm{C} 7$ & $0.0133(12)$ & $0.0144(12)$ & $0.0229(13)$ & $0.0005(10)$ & $-0.0015(10)$ & $-0.0027(10)$ \\
\hline $\mathrm{C} 8$ & $0.0154(13)$ & $0.0231(14)$ & $0.0307(15)$ & $-0.0027(11)$ & $-0.0060(11)$ & $0.0029(12)$ \\
\hline $\mathrm{C} 9$ & $0.0140(13)$ & $0.0151(12)$ & $0.0232(14)$ & $0.0007(10)$ & $0.0005(10)$ & $-0.0027(10)$ \\
\hline $\mathrm{C} 10$ & $0.0150(13)$ & $0.0198(13)$ & $0.0342(16)$ & $0.0016(11)$ & $-0.0037(12)$ & $0.0000(12)$ \\
\hline $\mathrm{C} 11$ & $0.0156(14)$ & $0.0202(14)$ & $0.0376(17)$ & $-0.0058(11)$ & $0.0060(12)$ & $0.0019(12)$ \\
\hline $\mathrm{C} 12$ & $0.0176(14)$ & $0.0169(13)$ & $0.0285(15)$ & $-0.0014(10)$ & $0.0049(11)$ & $0.0031(11)$ \\
\hline $\mathrm{C} 13$ & $0.0158(13)$ & $0.0134(12)$ & $0.0211(13)$ & $0.0004(10)$ & $0.0017(10)$ & $0.0001(10)$ \\
\hline $\mathrm{C} 14$ & 0.0185 (14) & $0.0136(12)$ & $0.0200(13)$ & $0.0010(10)$ & $0.0019(10)$ & $0.0007(10)$ \\
\hline $\mathrm{C} 15$ & 0.0204 (14) & $0.0265(15)$ & $0.0259(14)$ & $-0.0016(12)$ & $-0.0016(12)$ & $0.0095(12)$ \\
\hline $\mathrm{C} 16$ & $0.0172(13)$ & $0.0171(13)$ & $0.0202(13)$ & $-0.0036(10)$ & $-0.0024(10)$ & $0.0070(10)$ \\
\hline $\mathrm{C} 17$ & $0.0252(15)$ & $0.0243(14)$ & $0.0271(15)$ & $0.0012(12)$ & $0.0004(12)$ & $0.0013(12)$ \\
\hline $\mathrm{C} 18$ & $0.0180(15)$ & $0.0352(17)$ & $0.0423(18)$ & $0.0016(13)$ & $-0.0011(13)$ & $0.0077(15)$ \\
\hline C19 & $0.0189(15)$ & $0.0325(17)$ & $0.049(2)$ & $-0.0028(13)$ & $-0.0091(14)$ & $0.0144(15)$ \\
\hline $\mathrm{C} 20$ & $0.0326(17)$ & $0.0264(15)$ & $0.0385(18)$ & $-0.0112(13)$ & $-0.0160(14)$ & $0.0052(13)$ \\
\hline $\mathrm{C} 21$ & $0.0310(16)$ & $0.0194(14)$ & $0.0286(15)$ & $0.0013(12)$ & $-0.0071(13)$ & $0.0032(12)$ \\
\hline $\mathrm{Fe} 2$ & $0.0107(2)$ & $0.0120(2)$ & $0.0160(3)$ & $-0.0018(2)$ & 0.000 & 0.000 \\
\hline N4 & $0.0151(11)$ & $0.0149(10)$ & $0.0171(11)$ & $0.0001(8)$ & $-0.0001(9)$ & $0.0002(9)$ \\
\hline N5 & 0.0135 (11) & $0.0132(10)$ & $0.0171(11)$ & $-0.0028(8)$ & $-0.0001(8)$ & $0.0004(8)$ \\
\hline N6 & $0.0163(11)$ & $0.0145(10)$ & $0.0173(11)$ & $0.0005(9)$ & $0.0004(9)$ & $0.0000(9)$ \\
\hline $\mathrm{C} 22$ & $0.0166(13)$ & $0.0171(12)$ & $0.0157(12)$ & $-0.0052(10)$ & $0.0056(10)$ & $-0.0002(10)$ \\
\hline $\mathrm{C} 23$ & 0.0177 (13) & $0.0174(13)$ & $0.0212(14)$ & $-0.0019(11)$ & $0.0031(11)$ & $-0.0019(11)$ \\
\hline $\mathrm{C} 24$ & $0.0204(14)$ & $0.0246(14)$ & $0.0226(14)$ & $-0.0050(12)$ & $0.0012(11)$ & $-0.0014(12)$ \\
\hline $\mathrm{C} 25$ & $0.0259(15)$ & $0.0192(13)$ & $0.0257(14)$ & $-0.0047(12)$ & $0.0055(12)$ & $-0.0066(12)$ \\
\hline $\mathrm{C} 26$ & $0.0255(15)$ & 0.0154 (13) & $0.0277(15)$ & $0.0020(11)$ & $0.0117(12)$ & $-0.0027(11)$ \\
\hline $\mathrm{C} 27$ & $0.0184(13)$ & $0.0206(14)$ & $0.0211(13)$ & $-0.0019(11)$ & $0.0048(11)$ & $0.0012(11)$ \\
\hline $\mathrm{C} 28$ & 0.0087 (12) & 0.0189 (13) & $0.0212(13)$ & $-0.0001(10)$ & $0.0018(10)$ & $0.0008(11)$ \\
\hline $\mathrm{C} 29$ & $0.0168(14)$ & $0.0267(15)$ & $0.0320(16)$ & $-0.0031(11)$ & $0.0057(12)$ & $-0.0091(12)$ \\
\hline $\mathrm{C} 30$ & $0.0133(12)$ & $0.0171(12)$ & $0.0206(13)$ & $-0.0008(10)$ & $0.0011(10)$ & $0.0021(11)$ \\
\hline $\mathrm{C} 31$ & $0.0112(13)$ & $0.0215(14)$ & $0.0305(15)$ & $0.0008(11)$ & $0.0021(11)$ & $-0.0007(12)$ \\
\hline $\mathrm{C} 32$ & 0.0169 (14) & $0.0226(14)$ & $0.0327(16)$ & $-0.0058(11)$ & $-0.0046(12)$ & $-0.0011(12)$ \\
\hline $\mathrm{C} 33$ & $0.0230(14)$ & 0.0177 (13) & $0.0228(14)$ & $-0.0026(11)$ & $-0.0015(11)$ & $-0.0026(11)$ \\
\hline $\mathrm{C} 34$ & $0.0182(13)$ & $0.0135(12)$ & $0.0159(12)$ & $-0.0004(10)$ & $-0.0016(10)$ & $0.0019(10)$ \\
\hline C35 & $0.0172(13)$ & $0.0150(12)$ & $0.0163(12)$ & $0.0001(10)$ & $-0.0015(10)$ & $-0.0002(10)$ \\
\hline
\end{tabular}




\begin{tabular}{lllllll}
\hline & & & & & \\
C36 & $0.0194(14)$ & $0.0229(14)$ & $0.0246(14)$ & $-0.0020(11)$ & $0.0011(11)$ & $-0.0071(12)$ \\
C37 & $0.0174(13)$ & $0.0155(12)$ & $0.0204(13)$ & $-0.0019(10)$ & $0.0012(11)$ & $-0.0067(10)$ \\
C38 & $0.0186(15)$ & $0.0249(15)$ & $0.0358(17)$ & $-0.0002(11)$ & $0.0018(12)$ & $0.0015(13)$ \\
C39 & $0.0168(15)$ & $0.0318(17)$ & $0.059(2)$ & $0.0034(13)$ & $0.0021(15)$ & $-0.0043(16)$ \\
C40 & $0.0194(15)$ & $0.0300(17)$ & $0.059(2)$ & $-0.0070(13)$ & $0.0152(15)$ & $-0.0159(16)$ \\
C41 & $0.0331(17)$ & $0.0243(15)$ & $0.0399(18)$ & $-0.0087(13)$ & $0.0187(14)$ & $-0.0054(14)$ \\
C42 & $0.0226(15)$ & $0.0195(13)$ & $0.0280(15)$ & $0.0003(11)$ & $0.0067(12)$ & $-0.0030(12)$ \\
\hline
\end{tabular}

Geometric parameters $\left(\AA,{ }^{\circ}\right)$

\begin{tabular}{|c|c|c|c|}
\hline $\mathrm{Fe} 1-\mathrm{N} 1^{\mathrm{i}}$ & $2.149(2)$ & $\mathrm{Fe} 2-\mathrm{N} 4$ & $2.155(2)$ \\
\hline $\mathrm{Fe} 1-\mathrm{N} 1$ & $2.149(2)$ & $\mathrm{Fe} 2-\mathrm{N} 4^{\mathrm{ii}}$ & $2.155(2)$ \\
\hline $\mathrm{Fe} 1-\mathrm{N} 2$ & $2.028(2)$ & $\mathrm{Fe} 2-\mathrm{N} 5^{\mathrm{ii}}$ & $2.029(2)$ \\
\hline $\mathrm{Fe} 1-\mathrm{N} 2^{\mathrm{i}}$ & $2.028(2)$ & $\mathrm{Fe} 2-\mathrm{N} 5$ & $2.029(2)$ \\
\hline $\mathrm{Fe} 1-\mathrm{N} 3^{\mathrm{i}}$ & $2.173(2)$ & $\mathrm{Fe} 2-\mathrm{N}^{6 i}$ & $2.157(2)$ \\
\hline $\mathrm{Fe} 1-\mathrm{N} 3$ & $2.173(2)$ & $\mathrm{Fe} 2-\mathrm{N} 6$ & $2.157(2)$ \\
\hline $\mathrm{N} 1-\mathrm{C} 1$ & $1.418(3)$ & $\mathrm{N} 4-\mathrm{C} 22$ & $1.416(3)$ \\
\hline $\mathrm{N} 1-\mathrm{C} 7$ & $1.327(3)$ & $\mathrm{N} 4-\mathrm{C} 28$ & $1.327(3)$ \\
\hline N2-C9 & $1.368(3)$ & N5-C30 & $1.366(3)$ \\
\hline $\mathrm{N} 2-\mathrm{C} 13$ & $1.372(3)$ & $\mathrm{N} 5-\mathrm{C} 34$ & $1.368(3)$ \\
\hline N3-C14 & $1.300(3)$ & N6-C35 & $1.294(3)$ \\
\hline $\mathrm{N} 3-\mathrm{C} 16$ & $1.418(3)$ & N6-C37 & $1.422(3)$ \\
\hline $\mathrm{C} 1-\mathrm{C} 2$ & $1.396(4)$ & $\mathrm{C} 22-\mathrm{C} 23$ & $1.400(4)$ \\
\hline $\mathrm{C} 1-\mathrm{C} 6$ & $1.401(4)$ & $\mathrm{C} 22-\mathrm{C} 27$ & $1.397(4)$ \\
\hline $\mathrm{C} 2-\mathrm{H} 2$ & 0.9500 & $\mathrm{C} 23-\mathrm{H} 23$ & 0.9500 \\
\hline $\mathrm{C} 2-\mathrm{C} 3$ & $1.388(4)$ & $\mathrm{C} 23-\mathrm{C} 24$ & $1.381(4)$ \\
\hline $\mathrm{C} 3-\mathrm{H} 3$ & 0.9500 & $\mathrm{C} 24-\mathrm{H} 24$ & 0.9500 \\
\hline $\mathrm{C} 3-\mathrm{C} 4$ & $1.386(4)$ & $\mathrm{C} 24-\mathrm{C} 25$ & $1.390(4)$ \\
\hline $\mathrm{C} 4-\mathrm{H} 4$ & 0.9500 & $\mathrm{C} 25-\mathrm{H} 25$ & 0.9500 \\
\hline $\mathrm{C} 4-\mathrm{C} 5$ & $1.383(4)$ & $\mathrm{C} 25-\mathrm{C} 26$ & $1.385(4)$ \\
\hline $\mathrm{C} 5-\mathrm{H} 5$ & 0.9500 & $\mathrm{C} 26-\mathrm{H} 26$ & 0.9500 \\
\hline $\mathrm{C} 5-\mathrm{C} 6$ & $1.388(4)$ & $\mathrm{C} 26-\mathrm{C} 27$ & $1.395(4)$ \\
\hline C6-H6 & 0.9500 & $\mathrm{C} 27-\mathrm{H} 27$ & 0.9500 \\
\hline $\mathrm{C} 7-\mathrm{C} 8$ & $1.497(4)$ & $\mathrm{C} 28-\mathrm{C} 29$ & $1.502(4)$ \\
\hline $\mathrm{C} 7-\mathrm{C} 9$ & $1.443(4)$ & $\mathrm{C} 28-\mathrm{C} 30$ & $1.440(4)$ \\
\hline $\mathrm{C} 8-\mathrm{H} 8 \mathrm{~A}$ & 0.9800 & $\mathrm{C} 29-\mathrm{H} 29 \mathrm{~A}$ & 0.9800 \\
\hline $\mathrm{C} 8-\mathrm{H} 8 \mathrm{~B}$ & 0.9800 & $\mathrm{C} 29-\mathrm{H} 29 \mathrm{~B}$ & 0.9800 \\
\hline $\mathrm{C} 8-\mathrm{H} 8 \mathrm{C}$ & 0.9800 & $\mathrm{C} 29-\mathrm{H} 29 \mathrm{C}$ & 0.9800 \\
\hline $\mathrm{C} 9-\mathrm{C} 10$ & $1.401(4)$ & $\mathrm{C} 30-\mathrm{C} 31$ & $1.397(4)$ \\
\hline $\mathrm{C} 10-\mathrm{H} 10$ & 0.9500 & C $31-\mathrm{H} 31$ & 0.9500 \\
\hline $\mathrm{C} 10-\mathrm{C} 11$ & $1.382(4)$ & $\mathrm{C} 31-\mathrm{C} 32$ & $1.384(4)$ \\
\hline C11-H11 & 0.9500 & $\mathrm{C} 32-\mathrm{H} 32$ & 0.9500 \\
\hline $\mathrm{C} 11-\mathrm{C} 12$ & $1.390(4)$ & $\mathrm{C} 32-\mathrm{C} 33$ & $1.393(4)$ \\
\hline $\mathrm{C} 12-\mathrm{H} 12$ & 0.9500 & C $33-\mathrm{H} 33$ & 0.9500 \\
\hline $\mathrm{C} 12-\mathrm{C} 13$ & $1.381(4)$ & $\mathrm{C} 33-\mathrm{C} 34$ & $1.383(4)$ \\
\hline $\mathrm{C} 13-\mathrm{C} 14$ & $1.450(4)$ & C34-C35 & $1.456(4)$ \\
\hline $\mathrm{C} 14-\mathrm{C} 15$ & $1.505(4)$ & $\mathrm{C} 35-\mathrm{C} 36$ & $1.501(4)$ \\
\hline $\mathrm{C} 15-\mathrm{H} 15 \mathrm{~A}$ & 0.9800 & $\mathrm{C} 36-\mathrm{H} 36 \mathrm{~A}$ & 0.9800 \\
\hline
\end{tabular}




\begin{tabular}{|c|c|c|c|}
\hline C15-H15B & 0.9800 & $\mathrm{C} 36-\mathrm{H} 36 \mathrm{~B}$ & 0.9800 \\
\hline $\mathrm{C} 15-\mathrm{H} 15 \mathrm{C}$ & 0.9800 & $\mathrm{C} 36-\mathrm{H} 36 \mathrm{C}$ & 0.9800 \\
\hline $\mathrm{C} 16-\mathrm{C} 17$ & $1.389(4)$ & C37-C38 & $1.382(4)$ \\
\hline $\mathrm{C} 16-\mathrm{C} 21$ & $1.390(4)$ & $\mathrm{C} 37-\mathrm{C} 42$ & $1.391(4)$ \\
\hline C17-H17 & 0.9500 & C $38-\mathrm{H} 38$ & 0.9500 \\
\hline $\mathrm{C} 17-\mathrm{C} 18$ & $1.384(4)$ & $\mathrm{C} 38-\mathrm{C} 39$ & $1.386(4)$ \\
\hline C18-H18 & 0.9500 & C39-H39 & 0.9500 \\
\hline $\mathrm{C} 18-\mathrm{C} 19$ & $1.381(5)$ & $\mathrm{C} 39-\mathrm{C} 40$ & $1.382(5)$ \\
\hline C19-H19 & 0.9500 & $\mathrm{C} 40-\mathrm{H} 40$ & 0.9500 \\
\hline $\mathrm{C} 19-\mathrm{C} 20$ & $1.384(5)$ & $\mathrm{C} 40-\mathrm{C} 41$ & $1.387(5)$ \\
\hline $\mathrm{C} 20-\mathrm{H} 20$ & 0.9500 & $\mathrm{C} 41-\mathrm{H} 41$ & 0.9500 \\
\hline $\mathrm{C} 20-\mathrm{C} 21$ & $1.386(4)$ & $\mathrm{C} 41-\mathrm{C} 42$ & $1.383(4)$ \\
\hline $\mathrm{C} 21-\mathrm{H} 21$ & 0.9500 & $\mathrm{C} 42-\mathrm{H} 42$ & 0.9500 \\
\hline $\mathrm{N} 1-\mathrm{Fe} 1-\mathrm{N} 1^{\mathrm{i}}$ & $101.86(11)$ & $\mathrm{N} 4-\mathrm{Fe} 2-\mathrm{N} 4^{\mathrm{ii}}$ & $103.07(11)$ \\
\hline $\mathrm{N} 1{ }^{\mathrm{i}}-\mathrm{Fe} 1-\mathrm{N} 3$ & $90.40(8)$ & $\mathrm{N} 4-\mathrm{Fe} 2-\mathrm{N}^{\mathrm{ii}}$ & $89.87(8)$ \\
\hline $\mathrm{N} 1-\mathrm{Fe} 1-\mathrm{N} 3$ & $148.84(8)$ & $\mathrm{N} 4-\mathrm{Fe} 2-\mathrm{N} 6$ & $148.95(8)$ \\
\hline $\mathrm{N} 1-\mathrm{Fe} 1-\mathrm{N} 3^{\mathrm{i}}$ & $90.40(8)$ & $\mathrm{N} 4^{\mathrm{ii}}-\mathrm{Fe} 2-\mathrm{N} 6^{\mathrm{ii}}$ & $148.95(8)$ \\
\hline $\mathrm{N} 1^{\mathrm{i}}-\mathrm{Fe} 1-\mathrm{N} 3^{\mathrm{i}}$ & $148.84(8)$ & $\mathrm{N} 4{ }^{\mathrm{ii}}-\mathrm{Fe} 2-\mathrm{N} 6$ & $89.87(8)$ \\
\hline $\mathrm{N} 2-\mathrm{Fe} 1-\mathrm{N} 1^{\mathrm{i}}$ & $114.79(8)$ & $\mathrm{N} 5^{\mathrm{ii}}-\mathrm{Fe} 2-\mathrm{N} 4^{\mathrm{ii}}$ & $74.91(8)$ \\
\hline $\mathrm{N} 2 \mathrm{i}-\mathrm{Fe} 1-\mathrm{N} 1$ & $114.79(8)$ & $\mathrm{N} 5{ }^{\mathrm{ii}}-\mathrm{Fe} 2-\mathrm{N} 4$ & $113.41(8)$ \\
\hline $\mathrm{N} 2-\mathrm{Fe} 1-\mathrm{N} 1$ & $75.01(8)$ & $\mathrm{N} 5-\mathrm{Fe} 2-\mathrm{N} 4^{\mathrm{ii}}$ & $113.41(8)$ \\
\hline $\mathrm{N} 2^{\mathrm{i}}-\mathrm{Fe} 1-\mathrm{N} 1^{\mathrm{i}}$ & $75.01(8)$ & $\mathrm{N} 5-\mathrm{Fe} 2-\mathrm{N} 4$ & $74.91(8)$ \\
\hline $\mathrm{N} 2{ }^{\mathrm{i}}-\mathrm{Fe} 1-\mathrm{N} 2$ & $165.36(12)$ & $\mathrm{N} 55^{\mathrm{ii}}-\mathrm{Fe} 2-\mathrm{N} 5$ & $167.36(12)$ \\
\hline $\mathrm{N} 2-\mathrm{Fe} 1-\mathrm{N} 3^{\mathrm{i}}$ & $95.96(8)$ & $\mathrm{N} 5-\mathrm{Fe} 2-\mathrm{N}^{\mathrm{ii}}$ & $97.10(8)$ \\
\hline $\mathrm{N} 2-\mathrm{Fe} 1-\mathrm{N} 3$ & $73.84(8)$ & $\mathrm{N} 5-\mathrm{Fe} 2-\mathrm{N} 6$ & $74.04(8)$ \\
\hline $\mathrm{N} 2 \mathrm{i}-\mathrm{Fe} 1-\mathrm{N} 3$ & $95.96(8)$ & $\mathrm{N} 5^{\mathrm{ii}}-\mathrm{Fe} 2-\mathrm{N} 6^{\mathrm{ii}}$ & $74.05(8)$ \\
\hline $\mathrm{N} 2^{\mathrm{i}}-\mathrm{Fe} 1-\mathrm{N} 3^{\mathrm{i}}$ & $73.84(8)$ & $\mathrm{N} 5^{\mathrm{ii}}-\mathrm{Fe} 2-\mathrm{N} 6$ & $97.10(8)$ \\
\hline $\mathrm{N} 3^{\mathrm{i}}-\mathrm{Fe} 1-\mathrm{N} 3$ & $93.62(11)$ & $\mathrm{N} 6-\mathrm{Fe} 2-\mathrm{N} 6^{\mathrm{ii}}$ & $93.24(11)$ \\
\hline $\mathrm{C} 1-\mathrm{N} 1-\mathrm{Fe} 1$ & $121.09(16)$ & $\mathrm{C} 22-\mathrm{N} 4-\mathrm{Fe} 2$ & $120.83(16)$ \\
\hline $\mathrm{C} 7-\mathrm{N} 1-\mathrm{Fe} 1$ & $116.93(17)$ & $\mathrm{C} 28-\mathrm{N} 4-\mathrm{Fe} 2$ & $116.42(17)$ \\
\hline $\mathrm{C} 7-\mathrm{N} 1-\mathrm{C} 1$ & $121.5(2)$ & $\mathrm{C} 28-\mathrm{N} 4-\mathrm{C} 22$ & $122.5(2)$ \\
\hline $\mathrm{C} 9-\mathrm{N} 2-\mathrm{Fe} 1$ & $119.18(17)$ & $\mathrm{C} 30-\mathrm{N} 5-\mathrm{Fe} 2$ & $119.30(17)$ \\
\hline $\mathrm{C} 9-\mathrm{N} 2-\mathrm{C} 13$ & $119.0(2)$ & $\mathrm{C} 30-\mathrm{N} 5-\mathrm{C} 34$ & $119.0(2)$ \\
\hline $\mathrm{C} 13-\mathrm{N} 2-\mathrm{Fe} 1$ & $120.60(17)$ & $\mathrm{C} 34-\mathrm{N} 5-\mathrm{Fe} 2$ & $120.49(17)$ \\
\hline $\mathrm{C} 14-\mathrm{N} 3-\mathrm{Fe} 1$ & $117.66(18)$ & $\mathrm{C} 35-\mathrm{N} 6-\mathrm{Fe} 2$ & $118.10(18)$ \\
\hline $\mathrm{C} 14-\mathrm{N} 3-\mathrm{C} 16$ & $121.8(2)$ & $\mathrm{C} 35-\mathrm{N} 6-\mathrm{C} 37$ & $122.7(2)$ \\
\hline $\mathrm{C} 16-\mathrm{N} 3-\mathrm{Fe} 1$ & $120.47(16)$ & $\mathrm{C} 37-\mathrm{N} 6-\mathrm{Fe} 2$ & $119.16(16)$ \\
\hline $\mathrm{C} 2-\mathrm{C} 1-\mathrm{N} 1$ & $118.3(2)$ & $\mathrm{C} 23-\mathrm{C} 22-\mathrm{N} 4$ & $117.0(2)$ \\
\hline $\mathrm{C} 2-\mathrm{C} 1-\mathrm{C} 6$ & $118.3(2)$ & $\mathrm{C} 27-\mathrm{C} 22-\mathrm{N} 4$ & $124.7(2)$ \\
\hline $\mathrm{C} 6-\mathrm{C} 1-\mathrm{N} 1$ & $123.3(2)$ & $\mathrm{C} 27-\mathrm{C} 22-\mathrm{C} 23$ & $118.2(2)$ \\
\hline $\mathrm{C} 1-\mathrm{C} 2-\mathrm{H} 2$ & 119.7 & $\mathrm{C} 22-\mathrm{C} 23-\mathrm{H} 23$ & 119.4 \\
\hline $\mathrm{C} 3-\mathrm{C} 2-\mathrm{C} 1$ & $120.5(2)$ & $\mathrm{C} 24-\mathrm{C} 23-\mathrm{C} 22$ & $121.1(3)$ \\
\hline $\mathrm{C} 3-\mathrm{C} 2-\mathrm{H} 2$ & 119.7 & $\mathrm{C} 24-\mathrm{C} 23-\mathrm{H} 23$ & 119.4 \\
\hline $\mathrm{C} 2-\mathrm{C} 3-\mathrm{H} 3$ & 119.6 & $\mathrm{C} 23-\mathrm{C} 24-\mathrm{H} 24$ & 119.7 \\
\hline $\mathrm{C} 4-\mathrm{C} 3-\mathrm{C} 2$ & $120.8(3)$ & $\mathrm{C} 23-\mathrm{C} 24-\mathrm{C} 25$ & $120.5(3)$ \\
\hline $\mathrm{C} 4-\mathrm{C} 3-\mathrm{H} 3$ & 119.6 & $\mathrm{C} 25-\mathrm{C} 24-\mathrm{H} 24$ & 119.7 \\
\hline $\mathrm{C} 3-\mathrm{C} 4-\mathrm{H} 4$ & 120.4 & $\mathrm{C} 24-\mathrm{C} 25-\mathrm{H} 25$ & 120.6 \\
\hline
\end{tabular}




\begin{tabular}{|c|c|c|c|}
\hline $\mathrm{C} 5-\mathrm{C} 4-\mathrm{C} 3$ & $119.1(3)$ & $\mathrm{C} 26-\mathrm{C} 25-\mathrm{C} 24$ & $118.8(3)$ \\
\hline $\mathrm{C} 5-\mathrm{C} 4-\mathrm{H} 4$ & 120.4 & $\mathrm{C} 26-\mathrm{C} 25-\mathrm{H} 25$ & 120.6 \\
\hline $\mathrm{C} 4-\mathrm{C} 5-\mathrm{H} 5$ & 119.7 & $\mathrm{C} 25-\mathrm{C} 26-\mathrm{H} 26$ & 119.4 \\
\hline $\mathrm{C} 4-\mathrm{C} 5-\mathrm{C} 6$ & $120.7(3)$ & $\mathrm{C} 25-\mathrm{C} 26-\mathrm{C} 27$ & $121.1(3)$ \\
\hline $\mathrm{C} 6-\mathrm{C} 5-\mathrm{H} 5$ & 119.7 & $\mathrm{C} 27-\mathrm{C} 26-\mathrm{H} 26$ & 119.4 \\
\hline $\mathrm{C} 1-\mathrm{C} 6-\mathrm{H} 6$ & 119.7 & $\mathrm{C} 22-\mathrm{C} 27-\mathrm{H} 27$ & 120.0 \\
\hline $\mathrm{C} 5-\mathrm{C} 6-\mathrm{C} 1$ & $120.6(3)$ & $\mathrm{C} 26-\mathrm{C} 27-\mathrm{C} 22$ & $120.1(3)$ \\
\hline $\mathrm{C} 5-\mathrm{C} 6-\mathrm{H} 6$ & 119.7 & $\mathrm{C} 26-\mathrm{C} 27-\mathrm{H} 27$ & 120.0 \\
\hline $\mathrm{N} 1-\mathrm{C} 7-\mathrm{C} 8$ & $126.2(2)$ & $\mathrm{N} 4-\mathrm{C} 28-\mathrm{C} 29$ & $126.6(2)$ \\
\hline $\mathrm{N} 1-\mathrm{C} 7-\mathrm{C} 9$ & $114.0(2)$ & $\mathrm{N} 4-\mathrm{C} 28-\mathrm{C} 30$ & $114.4(2)$ \\
\hline $\mathrm{C} 9-\mathrm{C} 7-\mathrm{C} 8$ & $119.8(2)$ & $\mathrm{C} 30-\mathrm{C} 28-\mathrm{C} 29$ & $119.0(2)$ \\
\hline $\mathrm{C} 7-\mathrm{C} 8-\mathrm{H} 8 \mathrm{~A}$ & 109.5 & $\mathrm{C} 28-\mathrm{C} 29-\mathrm{H} 29 \mathrm{~A}$ & 109.5 \\
\hline $\mathrm{C} 7-\mathrm{C} 8-\mathrm{H} 8 \mathrm{~B}$ & 109.5 & $\mathrm{C} 28-\mathrm{C} 29-\mathrm{H} 29 \mathrm{~B}$ & 109.5 \\
\hline $\mathrm{C} 7-\mathrm{C} 8-\mathrm{H} 8 \mathrm{C}$ & 109.5 & $\mathrm{C} 28-\mathrm{C} 29-\mathrm{H} 29 \mathrm{C}$ & 109.5 \\
\hline $\mathrm{H} 8 \mathrm{~A}-\mathrm{C} 8-\mathrm{H} 8 \mathrm{~B}$ & 109.5 & $\mathrm{H} 29 \mathrm{~A}-\mathrm{C} 29-\mathrm{H} 29 \mathrm{~B}$ & 109.5 \\
\hline $\mathrm{H} 8 \mathrm{~A}-\mathrm{C} 8-\mathrm{H} 8 \mathrm{C}$ & 109.5 & $\mathrm{H} 29 \mathrm{~A}-\mathrm{C} 29-\mathrm{H} 29 \mathrm{C}$ & 109.5 \\
\hline $\mathrm{H} 8 \mathrm{~B}-\mathrm{C} 8-\mathrm{H} 8 \mathrm{C}$ & 109.5 & $\mathrm{H} 29 \mathrm{~B}-\mathrm{C} 29-\mathrm{H} 29 \mathrm{C}$ & 109.5 \\
\hline $\mathrm{N} 2-\mathrm{C} 9-\mathrm{C} 7$ & $114.2(2)$ & $\mathrm{N} 5-\mathrm{C} 30-\mathrm{C} 28$ & $114.1(2)$ \\
\hline $\mathrm{N} 2-\mathrm{C} 9-\mathrm{C} 10$ & $120.8(2)$ & $\mathrm{N} 5-\mathrm{C} 30-\mathrm{C} 31$ & $120.4(2)$ \\
\hline $\mathrm{C} 10-\mathrm{C} 9-\mathrm{C} 7$ & $125.0(2)$ & $\mathrm{C} 31-\mathrm{C} 30-\mathrm{C} 28$ & $125.4(2)$ \\
\hline $\mathrm{C} 9-\mathrm{C} 10-\mathrm{H} 10$ & 120.3 & $\mathrm{C} 30-\mathrm{C} 31-\mathrm{H} 31$ & 120.0 \\
\hline $\mathrm{C} 11-\mathrm{C} 10-\mathrm{C} 9$ & $119.5(3)$ & $\mathrm{C} 32-\mathrm{C} 31-\mathrm{C} 30$ & $120.0(3)$ \\
\hline $\mathrm{C} 11-\mathrm{C} 10-\mathrm{H} 10$ & 120.3 & $\mathrm{C} 32-\mathrm{C} 31-\mathrm{H} 31$ & 120.0 \\
\hline $\mathrm{C} 10-\mathrm{C} 11-\mathrm{H} 11$ & 120.1 & $\mathrm{C} 31-\mathrm{C} 32-\mathrm{H} 32$ & 120.1 \\
\hline $\mathrm{C} 10-\mathrm{C} 11-\mathrm{C} 12$ & $119.8(3)$ & $\mathrm{C} 31-\mathrm{C} 32-\mathrm{C} 33$ & $119.7(3)$ \\
\hline $\mathrm{C} 12-\mathrm{C} 11-\mathrm{H} 11$ & 120.1 & $\mathrm{C} 33-\mathrm{C} 32-\mathrm{H} 32$ & 120.1 \\
\hline $\mathrm{C} 11-\mathrm{C} 12-\mathrm{H} 12$ & 120.4 & $\mathrm{C} 32-\mathrm{C} 33-\mathrm{H} 33$ & 120.8 \\
\hline $\mathrm{C} 13-\mathrm{C} 12-\mathrm{C} 11$ & $119.1(2)$ & $\mathrm{C} 34-\mathrm{C} 33-\mathrm{C} 32$ & $118.4(2)$ \\
\hline $\mathrm{C} 13-\mathrm{C} 12-\mathrm{H} 12$ & 120.4 & $\mathrm{C} 34-\mathrm{C} 33-\mathrm{H} 33$ & 120.8 \\
\hline $\mathrm{N} 2-\mathrm{C} 13-\mathrm{C} 12$ & $121.8(2)$ & $\mathrm{N} 5-\mathrm{C} 34-\mathrm{C} 33$ & $122.4(2)$ \\
\hline $\mathrm{N} 2-\mathrm{C} 13-\mathrm{C} 14$ & $113.0(2)$ & $\mathrm{N} 5-\mathrm{C} 34-\mathrm{C} 35$ & $112.8(2)$ \\
\hline $\mathrm{C} 12-\mathrm{C} 13-\mathrm{C} 14$ & $125.1(2)$ & $\mathrm{C} 33-\mathrm{C} 34-\mathrm{C} 35$ & $124.6(2)$ \\
\hline $\mathrm{N} 3-\mathrm{C} 14-\mathrm{C} 13$ & $114.4(2)$ & $\mathrm{N} 6-\mathrm{C} 35-\mathrm{C} 34$ & $114.3(2)$ \\
\hline N3-C14-C15 & $124.7(2)$ & $\mathrm{N} 6-\mathrm{C} 35-\mathrm{C} 36$ & $125.1(2)$ \\
\hline $\mathrm{C} 13-\mathrm{C} 14-\mathrm{C} 15$ & $120.7(2)$ & $\mathrm{C} 34-\mathrm{C} 35-\mathrm{C} 36$ & $120.4(2)$ \\
\hline $\mathrm{C} 14-\mathrm{C} 15-\mathrm{H} 15 \mathrm{~A}$ & 109.5 & $\mathrm{C} 35-\mathrm{C} 36-\mathrm{H} 36 \mathrm{~A}$ & 109.5 \\
\hline $\mathrm{C} 14-\mathrm{C} 15-\mathrm{H} 15 \mathrm{~B}$ & 109.5 & $\mathrm{C} 35-\mathrm{C} 36-\mathrm{H} 36 \mathrm{~B}$ & 109.5 \\
\hline $\mathrm{C} 14-\mathrm{C} 15-\mathrm{H} 15 \mathrm{C}$ & 109.5 & $\mathrm{C} 35-\mathrm{C} 36-\mathrm{H} 36 \mathrm{C}$ & 109.5 \\
\hline $\mathrm{H} 15 \mathrm{~A}-\mathrm{C} 15-\mathrm{H} 15 \mathrm{~B}$ & 109.5 & $\mathrm{H} 36 \mathrm{~A}-\mathrm{C} 36-\mathrm{H} 36 \mathrm{~B}$ & 109.5 \\
\hline $\mathrm{H} 15 \mathrm{~A}-\mathrm{C} 15-\mathrm{H} 15 \mathrm{C}$ & 109.5 & $\mathrm{H} 36 \mathrm{~A}-\mathrm{C} 36-\mathrm{H} 36 \mathrm{C}$ & 109.5 \\
\hline $\mathrm{H} 15 \mathrm{~B}-\mathrm{C} 15-\mathrm{H} 15 \mathrm{C}$ & 109.5 & $\mathrm{H} 36 \mathrm{~B}-\mathrm{C} 36-\mathrm{H} 36 \mathrm{C}$ & 109.5 \\
\hline $\mathrm{C} 17-\mathrm{C} 16-\mathrm{N} 3$ & $119.8(2)$ & $\mathrm{C} 38-\mathrm{C} 37-\mathrm{N} 6$ & $120.5(2)$ \\
\hline $\mathrm{C} 17-\mathrm{C} 16-\mathrm{C} 21$ & $118.8(3)$ & $\mathrm{C} 38-\mathrm{C} 37-\mathrm{C} 42$ & $119.1(3)$ \\
\hline $\mathrm{C} 21-\mathrm{C} 16-\mathrm{N} 3$ & $121.0(3)$ & $\mathrm{C} 42-\mathrm{C} 37-\mathrm{N} 6$ & $120.1(2)$ \\
\hline $\mathrm{C} 16-\mathrm{C} 17-\mathrm{H} 17$ & 119.7 & $\mathrm{C} 37-\mathrm{C} 38-\mathrm{H} 38$ & 119.9 \\
\hline $\mathrm{C} 18-\mathrm{C} 17-\mathrm{C} 16$ & $120.7(3)$ & $\mathrm{C} 37-\mathrm{C} 38-\mathrm{C} 39$ & $120.3(3)$ \\
\hline $\mathrm{C} 18-\mathrm{C} 17-\mathrm{H} 17$ & 119.7 & $\mathrm{C} 39-\mathrm{C} 38-\mathrm{H} 38$ & 119.9 \\
\hline $\mathrm{C} 17-\mathrm{C} 18-\mathrm{H} 18$ & 119.8 & $\mathrm{C} 38-\mathrm{C} 39-\mathrm{H} 39$ & 119.6 \\
\hline
\end{tabular}




\begin{tabular}{|c|c|c|c|}
\hline $\mathrm{C} 19-\mathrm{C} 18-\mathrm{C} 17$ & $120.4(3)$ & $\mathrm{C} 40-\mathrm{C} 39-\mathrm{C} 38$ & $120.8(3)$ \\
\hline $\mathrm{C} 19-\mathrm{C} 18-\mathrm{H} 18$ & 119.8 & $\mathrm{C} 40-\mathrm{C} 39-\mathrm{H} 39$ & 119.6 \\
\hline $\mathrm{C} 18-\mathrm{C} 19-\mathrm{H} 19$ & 120.4 & $\mathrm{C} 39-\mathrm{C} 40-\mathrm{H} 40$ & 120.5 \\
\hline $\mathrm{C} 18-\mathrm{C} 19-\mathrm{C} 20$ & $119.2(3)$ & $\mathrm{C} 39-\mathrm{C} 40-\mathrm{C} 41$ & $119.0(3)$ \\
\hline $\mathrm{C} 20-\mathrm{C} 19-\mathrm{H} 19$ & 120.4 & $\mathrm{C} 41-\mathrm{C} 40-\mathrm{H} 40$ & 120.5 \\
\hline $\mathrm{C} 19-\mathrm{C} 20-\mathrm{H} 20$ & 119.6 & $\mathrm{C} 40-\mathrm{C} 41-\mathrm{H} 41$ & 119.8 \\
\hline $\mathrm{C} 19-\mathrm{C} 20-\mathrm{C} 21$ & $120.8(3)$ & $\mathrm{C} 42-\mathrm{C} 41-\mathrm{C} 40$ & $120.4(3)$ \\
\hline $\mathrm{C} 21-\mathrm{C} 20-\mathrm{H} 20$ & 119.6 & $\mathrm{C} 42-\mathrm{C} 41-\mathrm{H} 41$ & 119.8 \\
\hline $\mathrm{C} 16-\mathrm{C} 21-\mathrm{H} 21$ & 119.9 & $\mathrm{C} 37-\mathrm{C} 42-\mathrm{H} 42$ & 119.8 \\
\hline $\mathrm{C} 20-\mathrm{C} 21-\mathrm{C} 16$ & $120.1(3)$ & $\mathrm{C} 41-\mathrm{C} 42-\mathrm{C} 37$ & $120.5(3)$ \\
\hline $\mathrm{C} 20-\mathrm{C} 21-\mathrm{H} 21$ & 119.9 & $\mathrm{C} 41-\mathrm{C} 42-\mathrm{H} 42$ & 119.8 \\
\hline $\mathrm{Fe} 1-\mathrm{N} 1-\mathrm{C} 1-\mathrm{C} 2$ & $49.0(3)$ & $\mathrm{Fe} 2-\mathrm{N} 4-\mathrm{C} 22-\mathrm{C} 23$ & $-47.6(3)$ \\
\hline $\mathrm{Fe} 1-\mathrm{N} 1-\mathrm{C} 1-\mathrm{C} 6$ & $-126.8(2)$ & $\mathrm{Fe} 2-\mathrm{N} 4-\mathrm{C} 22-\mathrm{C} 27$ & $129.6(2)$ \\
\hline $\mathrm{Fe} 1-\mathrm{N} 1-\mathrm{C} 7-\mathrm{C} 8$ & $-178.0(2)$ & $\mathrm{Fe} 2-\mathrm{N} 4-\mathrm{C} 28-\mathrm{C} 29$ & $-177.2(2)$ \\
\hline $\mathrm{Fe} 1-\mathrm{N} 1-\mathrm{C} 7-\mathrm{C} 9$ & $-1.2(3)$ & $\mathrm{Fe} 2-\mathrm{N} 4-\mathrm{C} 28-\mathrm{C} 30$ & $4.7(3)$ \\
\hline $\mathrm{Fe} 1-\mathrm{N} 2-\mathrm{C} 9-\mathrm{C} 7$ & $9.7(3)$ & $\mathrm{Fe} 2-\mathrm{N} 5-\mathrm{C} 30-\mathrm{C} 28$ & $-8.9(3)$ \\
\hline $\mathrm{Fe} 1-\mathrm{N} 2-\mathrm{C} 9-\mathrm{C} 10$ & $-166.8(2)$ & $\mathrm{Fe} 2-\mathrm{N} 5-\mathrm{C} 30-\mathrm{C} 31$ & $168.2(2)$ \\
\hline $\mathrm{Fe} 1-\mathrm{N} 2-\mathrm{C} 13-\mathrm{C} 12$ & $167.8(2)$ & $\mathrm{Fe} 2-\mathrm{N} 5-\mathrm{C} 34-\mathrm{C} 33$ & $-169.7(2)$ \\
\hline $\mathrm{Fe} 1-\mathrm{N} 2-\mathrm{C} 13-\mathrm{C} 14$ & $-8.5(3)$ & $\mathrm{Fe} 2-\mathrm{N} 5-\mathrm{C} 34-\mathrm{C} 35$ & $6.0(3)$ \\
\hline $\mathrm{Fe} 1-\mathrm{N} 3-\mathrm{C} 14-\mathrm{C} 13$ & $-1.3(3)$ & $\mathrm{Fe} 2-\mathrm{N} 6-\mathrm{C} 35-\mathrm{C} 34$ & $2.6(3)$ \\
\hline $\mathrm{Fe} 1-\mathrm{N} 3-\mathrm{C} 14-\mathrm{C} 15$ & $-177.5(2)$ & $\mathrm{Fe} 2-\mathrm{N} 6-\mathrm{C} 35-\mathrm{C} 36$ & $178.1(2)$ \\
\hline $\mathrm{Fe} 1-\mathrm{N} 3-\mathrm{C} 16-\mathrm{C} 17$ & $-96.2(3)$ & $\mathrm{Fe} 2-\mathrm{N} 6-\mathrm{C} 37-\mathrm{C} 38$ & $96.8(3)$ \\
\hline $\mathrm{Fe} 1-\mathrm{N} 3-\mathrm{C} 16-\mathrm{C} 21$ & $76.4(3)$ & $\mathrm{Fe} 2-\mathrm{N} 6-\mathrm{C} 37-\mathrm{C} 42$ & $-75.9(3)$ \\
\hline $\mathrm{N} 1-\mathrm{C} 1-\mathrm{C} 2-\mathrm{C} 3$ & $-177.7(2)$ & $\mathrm{N} 4-\mathrm{C} 22-\mathrm{C} 23-\mathrm{C} 24$ & $-179.5(2)$ \\
\hline $\mathrm{N} 1-\mathrm{C} 1-\mathrm{C} 6-\mathrm{C} 5$ & $176.9(2)$ & $\mathrm{N} 4-\mathrm{C} 22-\mathrm{C} 27-\mathrm{C} 26$ & $-179.3(2)$ \\
\hline $\mathrm{N} 1-\mathrm{C} 7-\mathrm{C} 9-\mathrm{N} 2$ & $-5.2(3)$ & $\mathrm{N} 4-\mathrm{C} 28-\mathrm{C} 30-\mathrm{N} 5$ & $2.3(3)$ \\
\hline $\mathrm{N} 1-\mathrm{C} 7-\mathrm{C} 9-\mathrm{C} 10$ & $171.1(3)$ & $\mathrm{N} 4-\mathrm{C} 28-\mathrm{C} 30-\mathrm{C} 31$ & $-174.6(3)$ \\
\hline $\mathrm{N} 2-\mathrm{C} 9-\mathrm{C} 10-\mathrm{C} 11$ & $-0.5(4)$ & $\mathrm{N} 5-\mathrm{C} 30-\mathrm{C} 31-\mathrm{C} 32$ & $0.5(4)$ \\
\hline $\mathrm{N} 2-\mathrm{C} 13-\mathrm{C} 14-\mathrm{N} 3$ & $5.9(3)$ & $\mathrm{N} 5-\mathrm{C} 34-\mathrm{C} 35-\mathrm{N} 6$ & $-5.4(3)$ \\
\hline $\mathrm{N} 2-\mathrm{C} 13-\mathrm{C} 14-\mathrm{C} 15$ & $-177.7(2)$ & $\mathrm{N} 5-\mathrm{C} 34-\mathrm{C} 35-\mathrm{C} 36$ & $178.9(2)$ \\
\hline $\mathrm{N} 3-\mathrm{C} 16-\mathrm{C} 17-\mathrm{C} 18$ & $172.8(3)$ & $\mathrm{N} 6-\mathrm{C} 37-\mathrm{C} 38-\mathrm{C} 39$ & $-172.3(3)$ \\
\hline N3-C16-C21-C20 & $-172.3(3)$ & $\mathrm{N} 6-\mathrm{C} 37-\mathrm{C} 42-\mathrm{C} 41$ & $171.9(3)$ \\
\hline $\mathrm{C} 1-\mathrm{N} 1-\mathrm{C} 7-\mathrm{C} 8$ & $9.8(4)$ & $\mathrm{C} 22-\mathrm{N} 4-\mathrm{C} 28-\mathrm{C} 29$ & $-3.0(4)$ \\
\hline $\mathrm{C} 1-\mathrm{N} 1-\mathrm{C} 7-\mathrm{C} 9$ & $-173.4(2)$ & $\mathrm{C} 22-\mathrm{N} 4-\mathrm{C} 28-\mathrm{C} 30$ & $178.9(2)$ \\
\hline $\mathrm{C} 1-\mathrm{C} 2-\mathrm{C} 3-\mathrm{C} 4$ & $1.1(4)$ & $\mathrm{C} 22-\mathrm{C} 23-\mathrm{C} 24-\mathrm{C} 25$ & $-2.1(4)$ \\
\hline $\mathrm{C} 2-\mathrm{C} 1-\mathrm{C} 6-\mathrm{C} 5$ & $1.1(4)$ & $\mathrm{C} 23-\mathrm{C} 22-\mathrm{C} 27-\mathrm{C} 26$ & $-2.2(4)$ \\
\hline $\mathrm{C} 2-\mathrm{C} 3-\mathrm{C} 4-\mathrm{C} 5$ & $0.1(4)$ & $\mathrm{C} 23-\mathrm{C} 24-\mathrm{C} 25-\mathrm{C} 26$ & $-0.1(4)$ \\
\hline $\mathrm{C} 3-\mathrm{C} 4-\mathrm{C} 5-\mathrm{C} 6$ & $-0.8(4)$ & $\mathrm{C} 24-\mathrm{C} 25-\mathrm{C} 26-\mathrm{C} 27$ & $1.0(4)$ \\
\hline $\mathrm{C} 4-\mathrm{C} 5-\mathrm{C} 6-\mathrm{C} 1$ & $0.2(4)$ & $\mathrm{C} 25-\mathrm{C} 26-\mathrm{C} 27-\mathrm{C} 22$ & $0.1(4)$ \\
\hline $\mathrm{C} 6-\mathrm{C} 1-\mathrm{C} 2-\mathrm{C} 3$ & $-1.7(4)$ & $\mathrm{C} 27-\mathrm{C} 22-\mathrm{C} 23-\mathrm{C} 24$ & $3.2(4)$ \\
\hline $\mathrm{C} 7-\mathrm{N} 1-\mathrm{C} 1-\mathrm{C} 2$ & $-139.1(3)$ & $\mathrm{C} 28-\mathrm{N} 4-\mathrm{C} 22-\mathrm{C} 23$ & $138.5(3)$ \\
\hline $\mathrm{C} 7-\mathrm{N} 1-\mathrm{C} 1-\mathrm{C} 6$ & $45.1(4)$ & $\mathrm{C} 28-\mathrm{N} 4-\mathrm{C} 22-\mathrm{C} 27$ & $-44.3(4)$ \\
\hline $\mathrm{C} 7-\mathrm{C} 9-\mathrm{C} 10-\mathrm{C} 11$ & $-176.6(3)$ & $\mathrm{C} 28-\mathrm{C} 30-\mathrm{C} 31-\mathrm{C} 32$ & $177.3(3)$ \\
\hline $\mathrm{C} 8-\mathrm{C} 7-\mathrm{C} 9-\mathrm{N} 2$ & $171.9(2)$ & $\mathrm{C} 29-\mathrm{C} 28-\mathrm{C} 30-\mathrm{N} 5$ & $-175.9(2)$ \\
\hline $\mathrm{C} 8-\mathrm{C} 7-\mathrm{C} 9-\mathrm{C} 10$ & $-11.8(4)$ & $\mathrm{C} 29-\mathrm{C} 28-\mathrm{C} 30-\mathrm{C} 31$ & $7.2(4)$ \\
\hline $\mathrm{C} 9-\mathrm{N} 2-\mathrm{C} 13-\mathrm{C} 12$ & $0.7(4)$ & $\mathrm{C} 30-\mathrm{N} 5-\mathrm{C} 34-\mathrm{C} 33$ & $-2.5(4)$ \\
\hline $\mathrm{C} 9-\mathrm{N} 2-\mathrm{C} 13-\mathrm{C} 14$ & $-175.6(2)$ & $\mathrm{C} 30-\mathrm{N} 5-\mathrm{C} 34-\mathrm{C} 35$ & $173.2(2)$ \\
\hline
\end{tabular}




$\begin{array}{llll}\mathrm{C} 9-\mathrm{C} 10-\mathrm{C} 11-\mathrm{C} 12 & -0.8(4) & \mathrm{C} 30-\mathrm{C} 31-\mathrm{C} 32-\mathrm{C} 33 & -0.3(4) \\ \mathrm{C} 10-\mathrm{C} 11-\mathrm{C} 12-\mathrm{C} 13 & 2.0(4) & \mathrm{C} 31-\mathrm{C} 32-\mathrm{C} 33-\mathrm{C} 34 & -1.3(4) \\ \mathrm{C} 11-\mathrm{C} 12-\mathrm{C} 13-\mathrm{N} 2 & -1.9(4) & \mathrm{C} 32-\mathrm{C} 33-\mathrm{C} 34-\mathrm{N} 5 & 2.8(4) \\ \mathrm{C} 11-\mathrm{C} 12-\mathrm{C} 13-\mathrm{C} 14 & 173.9(3) & \mathrm{C} 32-\mathrm{C} 33-\mathrm{C} 34-\mathrm{C} 35 & -172.5(3) \\ \mathrm{C} 12-\mathrm{C} 13-\mathrm{C} 14-\mathrm{N} 3 & -170.2(3) & \mathrm{C} 33-\mathrm{C} 34-\mathrm{C} 35-\mathrm{N} 6 & 170.2(2) \\ \mathrm{C} 12-\mathrm{C} 13-\mathrm{C} 14-\mathrm{C} 15 & 6.2(4) & \mathrm{C} 33-\mathrm{C} 34-\mathrm{C} 35-\mathrm{C} 36 & -5.5(4) \\ \mathrm{C} 13-\mathrm{N} 2-\mathrm{C} 9-\mathrm{C} 7 & 177.1(2) & \mathrm{C} 34-\mathrm{N} 5-\mathrm{C} 30-\mathrm{C} 28 & -176.3(2) \\ \mathrm{C} 13-\mathrm{N} 2-\mathrm{C} 9-\mathrm{C} 10 & 0.6(4) & \mathrm{C} 34-\mathrm{N} 5-\mathrm{C} 30-\mathrm{C} 31 & 0.8(4) \\ \mathrm{C} 14-\mathrm{N} 3-\mathrm{C} 16-\mathrm{C} 17 & 79.8(3) & \mathrm{C} 35-\mathrm{N} 6-\mathrm{C} 37-\mathrm{C} 38 & -81.6(3) \\ \mathrm{C} 14-\mathrm{N} 3-\mathrm{C} 16-\mathrm{C} 21 & -107.5(3) & \mathrm{C} 35-\mathrm{N} 6-\mathrm{C} 37-\mathrm{C} 42 & 105.6(3) \\ \mathrm{C} 16-\mathrm{N} 3-\mathrm{C} 14-\mathrm{C} 13 & -177.5(2) & \mathrm{C} 37-\mathrm{N} 6-\mathrm{C} 35-\mathrm{C} 34 & -178.9(2) \\ \mathrm{C} 16-\mathrm{N} 3-\mathrm{C} 14-\mathrm{C} 15 & 6.3(4) & \mathrm{C} 37-\mathrm{N} 6-\mathrm{C} 35-\mathrm{C} 36 & -3.4(4) \\ \mathrm{C} 16-\mathrm{C} 17-\mathrm{C} 18-\mathrm{C} 19 & -0.7(5) & \mathrm{C} 37-\mathrm{C} 38-\mathrm{C} 39-\mathrm{C} 40 & 0.7(5) \\ \mathrm{C} 17-\mathrm{C} 16-\mathrm{C} 21-\mathrm{C} 20 & 0.5(4) & \mathrm{C} 38-\mathrm{C} 37-\mathrm{C} 42-\mathrm{C} 41 & -1.0(4) \\ \mathrm{C} 17-\mathrm{C} 18-\mathrm{C} 19-\mathrm{C} 20 & 1.0(5) & \mathrm{C} 38-\mathrm{C} 39-\mathrm{C} 40-\mathrm{C} 41 & -1.4(5) \\ \mathrm{C} 18-\mathrm{C} 19-\mathrm{C} 20-\mathrm{C} 21 & -0.6(5) & \mathrm{C} 39-\mathrm{C} 40-\mathrm{C} 41-\mathrm{C} 42 & 1.0(5) \\ \mathrm{C} 19-\mathrm{C} 20-\mathrm{C} 21-\mathrm{C} 16 & -0.2(5) & \mathrm{C} 40-\mathrm{C} 41-\mathrm{C} 42-\mathrm{C} 37 & 0.2(5) \\ \mathrm{C} 21-\mathrm{C} 16-\mathrm{C} 17-\mathrm{C} 18 & -0.1(4) & \mathrm{C} 42-\mathrm{C} 37-\mathrm{C} 38-\mathrm{C} 39 & 0.5(4) \\ \end{array}$

Symmetry codes: (i) $-x+1,-y+3 / 2, z$; (ii) $-x+1 / 2,-y+1, z$. 